\title{
Determination of complex aerodynamic admittance of bridge decks under deterministic gusts using the Vortex Particle Method
}

\author{
Igor Kavrakov ${ }^{\mathrm{a}, *}$, Tommaso Argentini ${ }^{\mathrm{b}}$, Simone Omarini $^{\mathrm{b}}$, Daniele Rocchi $^{\mathrm{b}}$, Guido Morgenthal $^{\mathrm{a}}$ \\ ${ }^{a}$ Chair of Modelling and Simulation of Structures, Bauhaus University Weimar, Marienstr. 13, Weimar 99423, Germany \\ ${ }^{b}$ Department of Mechanical Engineering, Politecnico di Milano, via La Masa 1, Milan 20156, Italy
}

\begin{abstract}
The accurate description of the aerodynamic forces due to free-stream turbulence acting on a stationary bridge deck represents a challenging task. This paper presents a Computational Fluid Dynamics (CFD) approach based on the two-dimensional (2D) Vortex Particle Method (VPM) for simulation of a six-component complex aerodynamic admittance. Deterministic free-stream turbulence is simulated by modeling the wakes of two fictitious pitching airfoils with vortex particles. For out-of- or in-phase sinusoidal oscillations of the airfoils, a longitudinal or vertical sinusoidal gust is obtained along the centerline, respectively. A closed-form solution, based on an existing mathematical model, is deduced to relate the gust amplitudes and vortex particles' circulation. Positioning a section downstream of the particle release locations yields sinusoidal buffeting forces. The complex aerodynamic admittance is then determined as a transfer function between the buffeting forces and the deterministic free-stream turbulence. A verification of the method is performed for the complex Sears' admittance of a flat plate. Finally, the CFD method is validated against wind tunnel tests for a streamlined bridge deck. The results from both, verification and validation, yielded a good agreement. Applications of the presented method are foreseen in the scope of buffeting analyses of line-like structures under the strip assumption.
\end{abstract}

Keywords: Aerodynamic admittance, Computational fluid dynamics, Vortex particle method, Buffeting, Long-span bridges

\section{Introduction}

The effect of the free-stream turbulence on the bluff bodies is perplexing and still not fully understood. A number of semianalytical models have been developed throughout the years to model the aerodynamic forces acting on a bridge deck due to free-stream wind gusts (buffeting forces). Perhaps, the most commonly utilized semi-analytical model in bridge aerodynamics is the linear unsteady model (Davenport, 1962; Diana et al., 2002; Chen and Kareem, 2002), which is based on the twodimensional (2D) linear unsteady airfoil theory and the strip assumption (Sears, 1941). In this model, the linear quasi-steady buffeting forces are modulated by an aerodynamic admittance, dependent on the chord-wise wavenumber, to account for the unsteady behavior. Therefore, the aerodynamic admittance represents a complex transfer function between the incoming wind gusts and aerodynamic forces.

Mainly, there are two approaches for experimental determination of the aerodynamic admittance in bridge aerodynamics. The first approach is based on random free-stream turbulence, generated either by grid or in boundary-layer wind tunnels (Larose, 2002; Gu and Qin, 2004). This approach considers the three-dimensional (3D) structure of the turbulence in a statistical manner through its power spectral density (PSD)

${ }^{*}$ Corresponding author. Tel. +49 (0) 3643584109

Email address: igor .kavrakov@uni-weimar .de (Igor Kavrakov) and coherence function. Thus, the absolute value of the aerodynamic admittance can be obtained as a transfer function between the PSDs of the wind fluctuations and measured buffeting forces. The second approach, which is of interest for this work, is based on deterministic free-stream turbulence in terms of sinusoidal gusts with a single prescribed frequency (Diana et al., 2002, 2013; Jancauskas and Melbourne, 1986; Han et al., 2010; Ma et al., 2013). There are number of ways of generating this type of gusts such as: an active turbulence generator (ATG) (Stapountzis and Graham, 1982; Harding et al., 2014; Argentini et al., 2012), circulation-controlled airfoils (Jancauskas and Melbourne, 1986), multiple fan arrangement (Ma et al., 2013; Cao et al., 2002), or rotating slotted cylinders (Tang et al., 1996). An ATG represents a set of two or more pitching airfoils, oscillating with a single frequency. For an out-ofor in-phase motion of two airfoils, longitudinal or vertical sinusoidal gusts are generated in the region between the wakes of the airfoils, respectively. The gusts generated in such way are considered to be fully correlated in the span-wise direction, which comply with the strip assumption. Positioning a deck section downstream of the ATG yields sinusoidal buffeting forces. Hence, this configuration allows to determine the real and imaginary part of the aerodynamic admittance as amplitude and phase modulation, respectively, between the sinusoidal buffeting forces and wind gusts.

Complementary to wind tunnel tests, methods based on Computational Fluid Dynamics (CFD) have gained a considerable momentum in the last two decades for gathering further insight 
in the physics of wind-bridge interaction. Unlike the static wind coefficients and flutter derivatives, the aerodynamic admittance has been rarely determined by utilizing CFD. To the authors' knowledge, very few studies exist that obtain the aerodynamic admittance with the grid-based CFD methods (e.g., Uejima et al. (2008); Turbelin and Gibert (2001); Bruno et al. (2004); Helgedagsurd et al. (2019)).

Alternative to the grid-based CFD methods, the 2D Vortex Particle Method (VPM) has found its application in bridge aerodynamics due to its low numerical dissipation and reasonable balance between resolved turbulent scales and computational cost (Ge and Xiang, 2008; Larsen and Walther, 1997; Kavrakov and Morgenthal, 2018a; Abbas et al., 2017; Kavrakov et al., 2019). Recently, Rasmussen et al. (2010) introduced a method for simulation of the $2 \mathrm{D}$ aerodynamic admittance for bridge decks based on the 2D VPM and synthetic random free-stream turbulence (Prendergast, 2007). Hejlesen et al. (2015) then utilized this method to obtain the aerodynamic admittance for several bridge decks, which were validated with experiments up to a certain extent. However, there are some limitations of this method, including the following: it cannot consider the aerodynamic admittance in its complex form; the contribution of the vortex-shedding and incident fluctuations to the buffeting forces are separated in an ad hoc manner (Kavrakov and Morgenthal, 2018b); and, the admittance for the longitudinal and vertical fluctuations are assumed to be similar. Moreover, this method may experience high numerical uncertainty as it involves mathematical operations with two PSDs of random processes.

Therefore, in this paper, we introduce a novel method based on the VPM and deterministic free-stream turbulence for simulation of the one-wavenumber six-component complex aerodynamic admittance. A free-stream sinusoidal gust is simulated by releasing vortex particles in the CFD domain, which constitute the wakes of two fictitious airfoils, and hence, a numerical ATG. Using the same analogy as in experiments, positioning a bridge section downstream of the ATG yields a complex aerodynamic admittance. Moreover, we deduce a closed-form solution to relate the particles' circulation and prescribed gust amplitudes. This solution is established on an existing analytical solution of an airfoil theory-based mathematical model (Stapountzis, 1982). The presented method is first verified with the complex analytical Sears' admittance and then compared to the Rassmusen's method based on random free-stream turbulence (Rasmussen et al., 2010). Finally, a validation is performed with experimental results from wind tunnel tests for the $3^{\text {rd }}$ Bosphours Bridge.

The article is organized as follows: The formulation of aerodynamic admittance for bridge deck is briefly outlined in Sec. 2 . In Sec. 3, we present the concept and individual components of the numerical ATG for simulation of a complex aerodynamic admittance. Section 4 entails verification of the flow field and aerodynamic admittance of a flat plate. The experimental validation for the $3^{\text {rd }}$ Bosphors bridge is given in Sec. 5. Section 6 constitutes critical remarks regarding the strip assumption and the aerodynamic admittance obtained using deterministic and random free-stream turbulence. Finally, concluding remarks are given in Sec. 7.

\section{Complex aerodynamic admittance}

The time-dependent aerodynamic forces acting at the stiffness center $\boldsymbol{x}_{s}=\left(x_{s}, 0\right)$ of a bluff body include a drag $D=D(t)$, lift $L=L(t)$ and moment $M=M(t)$ component (cf. Fig. 1), where $t$ denotes time. Generally, the aerodynamic forces can be normalized as follows:

$$
C_{D}^{*}=\frac{2 D}{\rho U^{2} B}, \quad C_{L}^{*}=\frac{2 L}{\rho U^{2} B}, \quad C_{M}^{*}=\frac{2 M}{\rho U^{2} B^{2}},
$$

where $\rho$ is the fluid density, $U$ is the mean wind velocity, $B$ is the body width, while $C_{D}^{*}=C_{D}^{*}(t), C_{L}^{*}=C_{L}^{*}(t)$, and $C_{M}^{*}=C_{M}^{*}(t)$ denote the drag, lift and moment fluctuating wind coefficients.

Based on their frequency content, the aerodynamic forces for a stationary body subjected to free-stream turbulence are characterized by the incident, shear layer (local or body-scale) and wake (vortex shedding) fluctuations (Kareem and Wu, 2013) The concept of the aerodynamic admittance depends on the model for the aerodynamic forces. In the 2D linear unsteady model, the aerodynamic forces for a stationary body are obtained as a linear superposition of the static and buffeting forces due to mean wind and incident fluctuations, respectively (Chen and Kareem, 2002). Further, the dependence on the turbulen$t$ characteristics in terms of intensity and the co-acting of the fluctuating components is not considered within this aerodynamic model (Ma et al., 2013; Matsuda et al., 1999; Zhao and Ge, 2015), as well as the local turbulence and vortex shedding effects. The aerodynamic forces per unit span then yield the following:

$$
D=D_{s}+D_{b}, \quad L=L_{s}+L_{b}, \quad M=M_{s}+M_{b},
$$

where the static and buffeting components are denoted with the subscripts " $s$ " and " $b$ ", respectively. The static forces due to mean wind are given as follows:

$$
D_{s}=\frac{1}{2} \rho U^{2} B C_{D}, \quad L_{s}=\frac{1}{2} \rho U^{2} B C_{L}, \quad M_{s}=\frac{1}{2} \rho U^{2} B^{2} C_{M},
$$

where $C_{D}=C_{D}\left(\alpha_{s}\right), C_{L}=C_{L}\left(\alpha_{s}\right)$, and $C_{M}=C_{M}\left(\alpha_{s}\right)$ are the drag lift and moment static wind coefficients, respectively. These coefficients are obtained from static wind tunnel tests by averaging and normalizing the aerodynamic forces at a static angle of attack $\alpha_{s}$. The buffeting forces are defined as follows:

$$
\begin{aligned}
D_{b} & =\frac{1}{2} \rho U^{2} B\left[2 C_{D} \chi_{D u} \frac{u}{U}+\left(C_{D}^{\prime}-C_{L}\right) \chi_{D w} \frac{w}{U}\right], \\
L_{b} & =\frac{1}{2} \rho U^{2} B\left[2 C_{L} \chi_{L u} \frac{u}{U}+\left(C_{L}^{\prime}+C_{D}\right) \chi_{L w} \frac{w}{U}\right], \\
M_{b} & =\frac{1}{2} \rho U^{2} B^{2}\left(2 C_{M} \chi_{M u} \frac{u}{U}+C_{M}^{\prime} \chi_{M w} \frac{w}{U}\right),
\end{aligned}
$$

where $u=u\left(\boldsymbol{x}_{c}, t\right)$ and $w=w\left(\boldsymbol{x}_{c}, t\right)$ are the longitudinal and vertical wind fluctuations, respectively, at a defined gust-tracking point $\boldsymbol{x}_{c} ; C_{D}^{\prime}=\mathrm{d} C_{D} / \mathrm{d} \alpha_{s}, C_{L}^{\prime}=\mathrm{d} C_{L} / \mathrm{d} \alpha_{s}$, and $C_{M}^{\prime}=\mathrm{d} C_{M} / \mathrm{d} \alpha_{s}$ are the derivatives of the static wind coefficients; and $\chi_{D u}, \chi_{D w}$, $\chi_{L u}, \chi_{L w}, \chi_{M u}$, and $\chi_{M w}$ are six complex aerodynamic admittance functions dependent on the reduced velocity $V_{r}=U /(f B)$, that is $\chi=\chi\left(V_{r}\right)$, where $f$ is the wind gust frequency. With 


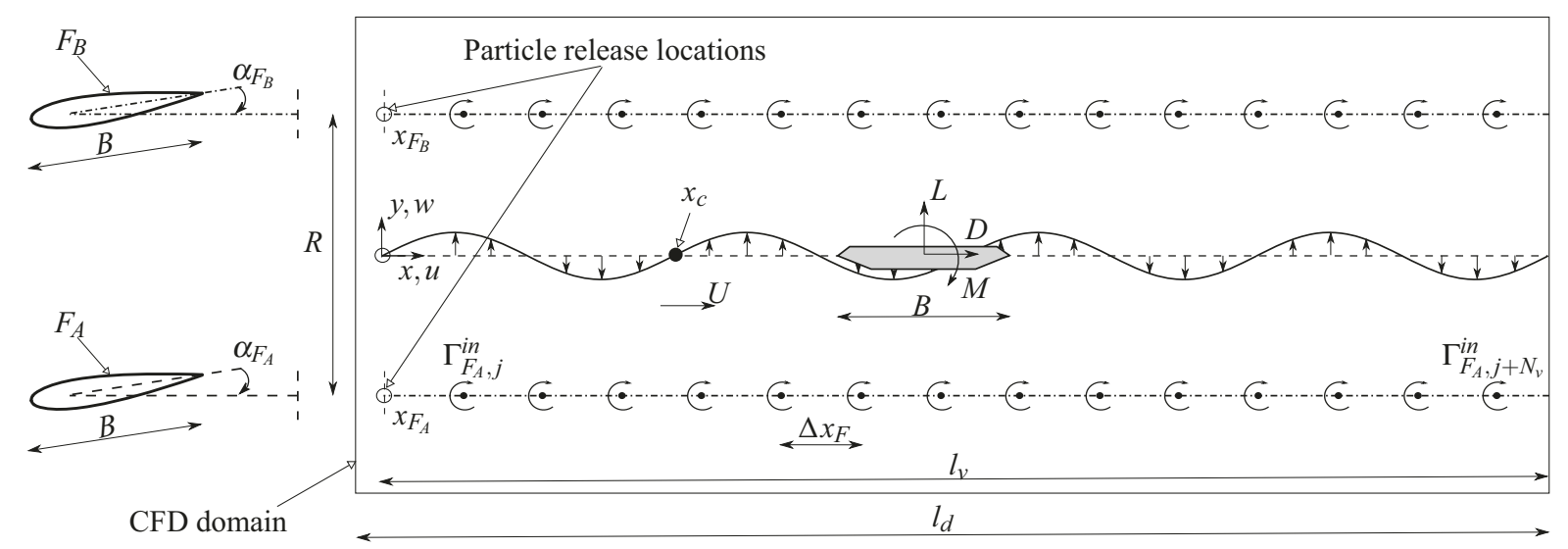

Figure 1: Concept for generation of deterministic free-stream turbulence for the determination of the complex aerodynamic admittance from a CFD analysis.

this formulation, the aerodynamic admittance is defined as a linear transfer function between the incident harmonic gusts and the aerodynamic forces, dependent only on the chord-wise wavenumber $k=\pi / V_{r}$. Since the aerodynamic forces are considered to be real signals, the aerodynamic admittance should take a complex form, that is, $\chi=F+i G$ (Kavrakov and Morgenthal, 2017). Here, $F$ and $G$ are the real and imaginary parts, respectively, corresponding the amplitude and phase modulation, respectively, while $i=\sqrt{-1}$ is the imaginary unit.

If we consider an incoming sinusoidal longitudinal $u=u_{0} \exp \left(i \omega_{t} t\right)$ or vertical gust $w=w_{0} \exp \left(i \omega_{t} t\right)$ with a corresponding amplitude $u_{0}$ or $w_{0}$ and prescribed circular frequency $\omega_{t}=2 \pi f_{t}$, it is straightforward to obtain the aerodynamic admittance. Taking the Fourier transform and rearranging Eqs. (4), the aerodynamic admittance functions yield the following:

$$
\begin{aligned}
& \chi_{D u}\left(V_{r t}\right)=\frac{\hat{D_{b}}\left(V_{r t}\right)}{\rho U B C_{D} \hat{u}\left(V_{r t}\right)}, \chi_{D w}\left(V_{r t}\right)=\frac{2 \hat{D_{b}}\left(V_{r t}\right)}{\rho U B\left(C_{D}^{\prime}-C_{L}\right) \hat{w}\left(V_{r t}\right)}, \\
& \chi_{L u}\left(V_{r t}\right)=\frac{\hat{L_{b}}\left(V_{r t}\right)}{\rho U B C_{L} \hat{u}\left(V_{r t}\right)}, \chi_{L w}\left(V_{r t}\right)=\frac{2 \hat{L_{b}}\left(V_{r t}\right)}{\rho U B\left(C_{L}^{\prime}+C_{D}\right) \hat{w}\left(V_{r t}\right)}, \\
& \chi_{M u}\left(V_{r t}\right)=\frac{\hat{M}_{b}\left(V_{r t}\right)}{\rho U B^{2} C_{M} \hat{u}\left(V_{r t}\right)}, \chi_{D w}\left(V_{r t}\right)=\frac{2 \hat{M}_{b}\left(V_{r t}\right)}{\rho U B^{2} C_{M}^{\prime} \hat{w}\left(V_{r t}\right)} .
\end{aligned}
$$

We denote the Fourier transform with a circumflex "^»" The subscripts " 0 " and " $t$ " denote an amplitude of a sinusoid and a prescribed (target) value, respectively. It is clear that the aerodynamic admittance can be obtained in its complex form from multiple analysis for various prescribed reduced velocities $V_{r t}=V_{r t}\left(f_{t}\right)$.

\section{Numerical active turbulence generator}

In this section, we initially describe the concept of a numerical ATG, based on the VPM, for determination of the complex aerodynamic admittance. Moreover, we briefly revisit the governing equations of the VPM. This is followed by an airfoil theory-based mathematical model to relate the particles' circulation and gust amplitudes.

\subsection{Concept}

Figure 1 depicts the concept of the presented method. Two fictitious airfoils, $F_{A}$ and $F_{B}$, are assumed to be oscillating upstream of the section and outside a CFD domain, constituting an ATG. The motion of the airfoils is rotational, sinusoidal and with similar amplitudes. The pitching angles for the two airfoils, $\alpha_{F_{A}}=\alpha_{F_{A}}(t)$ and $\alpha_{F_{B}}=\alpha_{F_{B}}(t)$, are given as follows:

$$
\alpha_{F_{A}}=\alpha_{F 0} \exp \left(i \omega_{t} t\right), \quad \alpha_{F_{B}}=\alpha_{F 0} \exp \left(i \omega_{t} t+i \phi\right),
$$

where $\alpha_{F 0}$ is the oscillation amplitude and $\phi$ is the phase between the motion of the two airfoils.

In the CFD domain, only the wakes of the airfoils are modeled by releasing particles carrying concentrated circulation $\Gamma_{F}^{i n}$. Once the particles are released in the CFD domain, their motion is governed by the Navier-Stokes equations, which are numerically solved utilizing the VPM. The particles are introduced at two particle release locations, $\boldsymbol{x}_{F_{A}}=(0,-R / 2)$ and $\boldsymbol{x}_{F_{B}}=(0, R / 2)$, at a constant time interval $\Delta t_{F}=\Delta p \Delta t$, where $\Delta p \in \mathbb{N}$ is the particle-release factor and $\Delta t$ is the simulation time-step. In case the oscillation of the airfoils is sinusoidal, the inflow discrete circulation for both foils, $\Gamma_{F_{A}}^{i n}=\Gamma_{F_{A}}^{i n}\left(\boldsymbol{x}_{F_{A}}, j\right)$ and $\Gamma_{F_{B}}^{\text {in }}=\Gamma_{F_{B}}^{\text {in }}\left(\boldsymbol{x}_{F_{B}}, j\right)$, are also sinusoidal and are given as follows:

$$
\Gamma_{F_{A}, j}^{i n}=\Gamma_{F 0}^{i n} \exp \left(i \omega_{t} j \Delta t_{F}\right), \quad \Gamma_{F_{B}, j}^{i n}=\Gamma_{F 0}^{i n} \exp \left(i \omega_{t} j \Delta t_{F}+i \phi\right),
$$

where $j=\left\{1, \ldots,\left\lfloor N_{s} / \Delta p\right\rfloor\right\}$, for $N_{s} \in \mathbb{N}$ being number of simulation steps. The circulation amplitude depends on the temporal discretization, that is, $\Gamma_{F 0}=\Gamma_{F 0}\left(\Delta t_{F}\right)$. Assuming Taylor's hypothesis holds, the spatial and temporal discretization are related as $\Delta x_{F}=U \Delta t_{F}$, where $\Delta x_{F}$ is the particle spacing.

If $\Gamma_{F_{A}}^{i n}$ and $\Gamma_{F_{B}}^{i n}$ are in-phase $(\phi=0)$ or out-of-phase $(\phi=\pi)$, a vertical or horizontal sinusoidal gust is obtained, respectively, at a gust-tracking point on the centerline, that is, $\boldsymbol{x}_{c}=\left(x_{c}, 0\right)$. However, this is only true if the following assumptions hold: 
i) the vorticity shed from an airfoil into the wake is concentrated at the mean chord line and is convected by the mean velocity (planar wake assumption);

ii) the wakes are non-interfering and infinite, and the point $\boldsymbol{x}_{c}$ is located sufficiently downstream of the particle release locations (or the airfoils).

We note that these assumptions are required for a gust at point $\boldsymbol{x}_{c}$ along the centerline to be sinusoidal. As discussed later in Sec. 4, these do not always hold in the CFD analyses; hence, there is a deviation from a pure sinusoidal gust. In order to relate the pitching angle amplitude of the airfoils $\alpha_{F 0}$ to the circulation amplitude $\Gamma_{F 0}$, and hence, to prescribed gust amplitudes $u_{t 0}$ or $w_{t 0}$, further assumptions are required as discussed further in the subsequent sections.

Positioning a section downstream of the particle release locations results in sinusoidal aerodynamic forces. Hence, the aerodynamic admittance can be computed straight-forward (cf. Eqs. (5)). In light of the previous explanation, the presented method consists of two essential steps and one optional step, in which:

a) the released circulation $\Gamma_{F}^{\text {in }}$ (cf. Eqs. (7)) is computed a-priori of the CFD simulations for a prescribed gust amplitude, $u_{t 0}$ or $w_{t 0}$, and frequency $f_{t}$;

b) a CFD simulation with a stationary body is conducted including upstream released particles;

c) (optional) a CFD simulation without a body is conducted including upstream released particles.

The optional step is included in case the gust-tracking point $\boldsymbol{x}_{c}$ is selected to be at the stiffness center, i.e. $\boldsymbol{x}_{c} \equiv \boldsymbol{x}_{s}$. In this case, the effect of section on the incoming gust is not taken into account and the imaginary part of the admittance is obtained for wind fluctuations at the stiffness center, which is the situation for the linear unsteady model based on the airfoil theory.

Modeling flapping airfoils using concentrated vortices has been used in a form of mathematical models, based on airfoil theory (cf. e.g. Stapountzis (1982); Harding and Bryden (2012)), to determine the airfoil flapping angles w.r.t. gust amplitudes. Chawdhury et al. (2018) used the concept of counterrotating vortices in the VPM to simulate pulsating flow, i.e. longitudinal gusts with much longer lengths than the CFD domain, to study the performance of flutter-based energy harvesters. Herein, the circulation amplitude of the released particles $\Gamma_{F 0}^{i n}$ for a prescribed gust amplitude, $u_{c t 0}$ or $w_{c t 0}$, in step a) is obtained from an airfoil theory-based mathematical model (Stapountzis, 1982), as shown in the sequel. It is noteworthy to mention that, the prescribed gust amplitude in step a) is only to provide an approximate value, which will be later corrected by the one tracked in the CFD domain within steps b) or c). The prescribed gust amplitude should also ensure that, up to a certain extent, the aerodynamic forces remain linear.

\subsection{Vortex particle method}

Considering a Newtonian fluid, the 2D Navier-Stokes equations in the vorticity transport form are given as follows (Cottet and Koumoutsakos, 2000; Morgenthal and Walther, 2007):

$$
\frac{\partial \omega_{u}}{\partial t}+(\boldsymbol{u} \cdot \nabla) \omega_{u}=v \nabla^{2} \omega_{u}
$$

where $\boldsymbol{u}=(u, w, 0)$ is the velocity vector. The vorticity is a scalar in $2 \mathrm{D}$, that is, $\omega_{u}=\left(0,0, \omega_{u}\right)$, and is related to the velocity as follows $\omega_{u}=\omega_{u}(x, t)=\nabla \times u$, where $v$ is the kinematic viscosity. For an incompressible fluid, that is, $\nabla \cdot \boldsymbol{u}=0$, the velocity field is obtained from the vorticity field by solving the inverted kinematic relation (Hejlesen et al., 2015):

$$
\nabla^{2} \boldsymbol{u}=-\nabla \times \omega_{u} .
$$

The preceding equation represents a Poission equation, which can be solved using Green's function, resulting in the BiotSavart's law as follows:

$$
\boldsymbol{u}(\boldsymbol{x})=\boldsymbol{U}-\frac{1}{2 \pi} \int_{\mathcal{D}} \frac{(\boldsymbol{x}-\boldsymbol{y}) \times \omega_{u}(\boldsymbol{y})}{|\boldsymbol{x}-\boldsymbol{y}|^{2}} \mathrm{~d} \boldsymbol{y},
$$

where $\boldsymbol{U}=(U, 0,0)$ is the free-stream velocity vector and $\mathcal{D}$ is the domain including the fluid and body.

The vorticity is the property which is advanced in Eq. (8). In the VPM, the vorticity field is discretized by vortex particles characterized by their locations $\boldsymbol{x}_{p}=\left(x_{p}, y_{p}, 0\right)$ and strength $\boldsymbol{\Gamma}_{p}=\left(0,0, \Gamma_{p}\right)$. Their strength (i.e. circulation) is obtained by integrating the vorticity over a small patch of fluid $\mathcal{D}_{p}$ as $\Gamma_{p}=\int_{\mathcal{D}_{p}} \omega_{u}(\boldsymbol{x}) \mathrm{d} \mathcal{D}_{p}$. Taking this into account, the discrete form of the Biot-Savart's relation (cf. Eq. (10)), in a Eulerian frame of reference, is given as follows:

$$
\boldsymbol{u}\left(\boldsymbol{x}_{p}\right)=\boldsymbol{U}-\frac{1}{2 \pi} \sum_{p=1}^{N_{p}} \frac{\left(\boldsymbol{x}-\boldsymbol{x}_{p}\right) \times \boldsymbol{\Gamma}_{p}}{\left|\boldsymbol{x}-\boldsymbol{x}_{p}\right|^{2}}=\boldsymbol{U}-\sum_{p=1}^{N_{p}} \boldsymbol{K} \Gamma_{p} .
$$

where $\boldsymbol{K}=\boldsymbol{K}\left(\boldsymbol{x}-\boldsymbol{x}_{p}\right)$ is referred to as a velocity kernel, which is substituted by a mollified velocity kernel $\boldsymbol{K}_{\epsilon}$ to account for the numerical instabilities if $\boldsymbol{x}-\boldsymbol{x}_{p} \rightarrow 0$.

In the numerical implementation, the vorticity transport equation (cf. Eq. (8)) is solved in two steps using the operator spitting technique (Chorin, 1973). Within this tehnique, the convection and diffusion are solved sequentially. In the convection step, the advancement of the inviscid Euler equation is approximated by convection of the vortex particles utilizing the standard Runge-Kutta time-marching techniques. The kinematics of the particles is obtained by solving the Poisson equation (cf. Eq. (9)) on a regularized grid and Eq. (11) for the sub-grid particle velocities. Numerically, the kinematics are solved utilizing the Vortex-In-Cell algorithms (Hockney and Eastwood, 1988) based on the fast Fourier transform (FFT). Herein, the efficient $\mathrm{P}^{3} \mathrm{M}$ algorithm by Morgenthal and Walther (2007) is employed. The random walk method by Chorin (1973) is used for the diffusion step. With this method, the particles' position $\boldsymbol{x}_{p}$ is petributed in a random direction with a random magnitude with zero mean and $2 v \Delta t$ variance.

For the discretization of an immersed body, the boundary element method is utilized ( $\mathrm{Wu}, 1976)$. Within this method, the geometry of the body is discretized on finite number of panels, at which surface there is a linear variation of the surface 
vortex sheets. The no-slip and no-penetration velocity boundary conditions are implicitly enforced by imposing the vorticity boundary conditions, and additionally, Kelvin's circulation theorem to yield a unique solution (Walther and Larsen, 1997). It is important to note that Kelvin's circulation theorem is modified to account for the released particles as follows (Prendergast, 2007):

$$
\sum \Gamma=\sum\left(\Gamma_{F_{A}}^{i n}+\Gamma_{F_{B}}^{i n}\right)
$$

Finally, the aerodynamic forces are obtained by integrating the surface pressure, obtained from the surface vorticity.

Further discussions on the VPM are ommited herein for the sake of brevity. For detailed information, we refer to Cottet and Koumoutsakos (2000) for the governing equations of the VPM and to Morgenthal and Walther (2007); Morgenthal et al. (2014) for the numerical implementation utilized in this work.

\subsection{Mathematical model}

The mathematical model, used to determine circulation amplitude $\Gamma_{F 0}^{i n}$ (cf. Eqs. (7)) for a prescribed gust amplitude, practically enforces the two assumptions in Sec. 3.1. Furthermore, two additional assumptions are required since the wakes' are modeled utilizing the airfoil theory, namely:

iii) the airfoils are idealized as flat plates and the oscillation amplitudes are sufficiently small, so that linear unsteady theory applies;

iv) the Kutta-Jukowski condition is constantly fulfilled.

For an oscillating airfoil, which trailing edge is positioned at $\left(0, y_{F}\right)$, the induced velocity fluctuations at a point on the centerline $\boldsymbol{x}_{c}$ can be obtained by using the Biot-Savart's law (cf. Eq. (10)) as follows:

$$
\begin{aligned}
& u_{c}^{F}=-\frac{y_{F}}{2 \pi} \int_{0}^{\infty} \frac{\gamma_{F}(x)}{\left(x_{c}-x\right)^{2}+y^{2}} \mathrm{~d} x, \\
& w_{c}^{F}=-\frac{1}{2 \pi} \int_{0}^{\infty} \frac{\left(x_{c}-x\right) \gamma_{F}(x)}{\left(x_{c}-x\right)^{2}+y_{F}^{2}} \mathrm{~d} x,
\end{aligned}
$$

where, $\gamma_{F}=\gamma_{F}\left(\boldsymbol{x}_{\gamma}, t\right)$ is the wake vorticity of the airfoil at point $\boldsymbol{x}_{\gamma}=\left(x, y_{F}\right)$, which is concentrated along the line $y=y_{F}$ (cf. assumption (i) from Sec. 3.1). Assumption (ii) from Sec. 3.1 is implied in Eqs. (13) by neglecting the bound circulation, as the point $\boldsymbol{x}_{c}$ is assumed to be sufficiently downstream.

For two oscillating airfoils with non-interfering wakes, the velocity components at the centerline are obtained as follows:

$$
u_{c}=u_{c}^{F_{A}}+u_{c}^{F_{B}}, \quad w_{c}=w_{c}^{F_{A}}+w_{c}^{F_{B}} .
$$

To obtain a solution for Eqs. (13) and (14), we use two methods to which we will refer to as "Analytical" and "Inverse" method.

\subsubsection{Analytical method}

For an airfoil performing sinusoidal rotation with prescribed frequency $\omega_{t}$, the wake vorticity is also sinusoidal
$\gamma_{F}=\gamma_{F 0} \exp \left(i \omega_{t} t\right)$. Stapountzis $(1978,1982)$ gives a closedform solution of Eqs. (13), based on the assumption (i-iv), as follows:

$$
\begin{aligned}
& u_{c}^{F}=-\operatorname{sgn}\left(y_{F}\right) \frac{S}{2} \exp \left[-\frac{2 k_{t}}{B}\left|y_{F}\right|+i\left(\omega_{t} t-\frac{2 k_{t}}{B} x_{c}\right)\right], \\
& w_{c}^{F}=i \frac{S}{2} \exp \left[-\frac{2 k_{t}}{B}\left|y_{F}\right|+i\left(\omega_{t} t-\frac{2 k_{t}}{B} x_{c}\right)\right],
\end{aligned}
$$

where sgn is the sign function, $k_{t}=\omega_{t} B /(2 U)$ and $S=S\left(k_{t}\right)$ is given as follows:

$$
S=-4 U \alpha_{F 0} \frac{1+(0.5-2 m) i k_{t}}{H_{1}^{(2)}+i H_{0}^{(2)}} .
$$

where $H_{1}^{(2)}=H_{1}^{(2)}\left(k_{t}\right)$ and $H_{0}^{(2)}=H_{0}^{(2)}\left(k_{t}\right)$ are Hankel functions of the second kind. The non-dimensional distance between the the rotation center and mid-chord point of the airfoil is defined by the parameter $m$.

In the present configuration, two airfoils are positioned at $\boldsymbol{x}_{F_{A}}$ and $\boldsymbol{x}_{F_{B}}$ (cf. Fig. 1). The velocities at the centerline can be obtained by changing Eqs. (15) into Eqs. (14). For in-phase oscillations of the airfoils, $u_{c}^{F_{A}}=-u_{c}^{F_{B}}$ and $w_{c}^{F_{A}}=w_{c}^{F_{B}}$, hence the velocities at the centerline yield the following:

$$
u_{c}=0, \quad w_{c}=i S \exp \left[-\frac{k_{t} R}{B}+i\left(\omega_{t} t-\frac{2 k_{t}}{B} x_{c}\right)\right] .
$$

For out-of-phase oscillations of the airfoils, $u_{c}^{F_{A}}=u_{c}^{F_{B}}$ and $w_{c}^{F_{A}}=-w_{c}^{F_{B}}$; hence, the velocities at the centerline are obtained as follows:

$$
u_{c}=-S \exp \left[-\frac{k_{t} R}{B}+i\left(\omega_{t} t-\frac{2 k_{t}}{B} x_{c}\right)\right], \quad w_{c}=0 .
$$

In the experimental studies (Stapountzis and Graham, 1982) the aim is to have a closed-form solution that relates the oscillation amplitude of the airfoils $\alpha_{F 0}$ to the gust amplitudes $w_{c 0}$ and $u_{c 0}$. To relate the gust amplitude to the circulation in a closedform solution, we deduce the following equations in sequel.

First, consider the case for in-phase oscillating airfoils, that is, $\Gamma_{F_{A}}^{i n}=\Gamma_{F_{B}}^{i n}=\Gamma_{F}^{i n}$. For airfoils oscillating about the front quarter-point, the parameter $m$ amounts to $m=-0.25$. Taking this into account, changing Eq. (16) in Eq. (17) for the vertical velocity and using the non-dimensional time $s=t U / B$, the following is obtained:

$$
\alpha_{F 0}=-\frac{w_{c}\left(H_{1}^{(2)}+i H_{0}^{(2)}\right)}{4 U\left(1+i k_{t}\right) i} \exp \left[\frac{k_{t} R}{B}-i\left(2 k_{t} s-\frac{2 k_{t}}{B} x_{c}\right)\right] .
$$

For a sinusoidal rotational motion of the airfoils, the vertical velocity is also sinusoidal, that is, $w_{c}=w_{c}(s)=w_{c 0} \exp \left(i k_{t} s\right)$. Furthermore, the circulatory lift deficiency of such airfoils due to the unsteady behavior is accounted for by replacing the angle of oscillation $\alpha_{F}$ (cf. Eqs. (6)) with the effective angle $\alpha_{e}=\alpha_{e}(s)=\alpha_{F} / C$ in the quasi-steady formulation for the lift (Bisplinghoff et al., 1996). Changing this in Eq. (19), yields the following:

$$
\alpha_{e}=-C \frac{w_{c 0}\left(H_{1}^{(2)}+i H_{0}^{(2)}\right)}{4 U\left(1+i k_{t}\right) i} \exp \left[\frac{k_{t} R}{B}-i\left(2 k_{t} s-\frac{2 k_{t}}{B} x_{c}\right)\right],
$$


where $C=C\left(k_{t}\right)$ is the Theodorsen's circulatory function, which given as follows:

$$
C=\frac{H_{1}^{(2)}}{H_{1}^{(2)}+i H_{0}^{(2)}} .
$$

The bound circulation $\Gamma_{b}=\Gamma_{b}(s)$ is obtained based on the Kutta-Jukowski theorem and the effective angle as $\Gamma_{b}=$ $U \pi \alpha_{e} B$. Taking this into account and changing Eq. (21) into Eq. (20), the following expression is obtained:

$$
\Gamma_{b}=\frac{w_{c 0} \pi B H_{1}^{(2)}}{4\left(1+i k_{t}\right) i} \exp \left(\frac{k_{t} R}{B}+i 2 k_{t} s\right)
$$

In the preceding equation, the sign, the term $2 k_{t} / B x_{c}$ and sign under the exponential in Eq. (20) are neglected. These terms account for the phase between the circulation at the particle release locations and velocity fluctuations at point $\boldsymbol{x}_{c}$. This phase is obsolete for the purpose of this paper.

The discrete circulation shed in the wake due to change of angle of the airfoil during time $\Delta s_{F}=\Delta t_{F} U / B$ is $\Gamma_{F}^{i n}=\Gamma_{F}^{i n}(s)=$ $\Gamma_{b}\left(s-\Delta s_{F}\right)-\Gamma_{b}(s)$. Taking this into account and discretizing the non-dimensional time as $s=j \Delta s$, the following relation is obtained:

$\Gamma_{F, j}^{i n}=\frac{w_{c 0} \pi B H_{1}^{(2)}}{4\left(1+i k_{t}\right) i} \exp \left(\frac{k_{t} R}{B}\right)\left[\exp \left(-i 2 k_{t} \Delta s_{F}\right)-1\right] \exp \left(i 2 k_{t} j \Delta s_{F}\right)$

Similarly, for the out-of-phase motion in Eq. (18), that is $\Gamma_{F_{A}}^{i n}=\Gamma_{F}^{i n}$ and $\Gamma_{F_{B}}^{i n}=-\Gamma_{F}^{i n}$, the following expression is obtained:

$\Gamma_{F, j}^{i n}=\frac{u_{c 0} \pi B H_{1}^{(2)}}{4\left(1+i k_{t}\right)} \exp \left(\frac{k_{t} R}{B}\right)\left[\exp \left(-i 2 k_{t} \Delta s_{F}\right)-1\right] \exp \left(i 2 k_{t} j \Delta s_{F}\right)$.

Both, Eqs. (23) and (24), represent the relation between the released circulation $\Gamma_{F}^{i n}$ and gust amplitudes for in-phase and out-of-phase motion of the airfoils. Both equations can be reduced in the following form:

$$
\Gamma_{F, j}^{i n}=\Gamma_{F 0}^{i n} \exp \left(i 2 k_{t} j \Delta s_{F}\right)
$$

which is similar to Eq. (7) for non-dimensional time. The amplitude of the circulation is the same for vertical or longitudinal sinusoidal gusts with similar prescribed amplitudes $u_{c t 0}=w_{c t 0}$. Neglecting the phase, the final closed-form solution for the circulation amplitude $\Gamma_{F 0}^{i n}=\Gamma_{F 0}^{i n}\left(\Delta s_{F}\right)$ yields the following:

$$
\begin{aligned}
\Gamma_{F 0}^{i n} & =\left|\frac{w_{c t 0} \pi B H_{1}^{(2)}}{4\left(1+i k_{t}\right) i} \exp \left(\frac{k_{t} R}{B}\right)\left[\exp \left(-i 2 k_{t} \Delta s_{F}\right)-1\right]\right| \\
& =\left|\frac{u_{c t 0} \pi B H_{1}^{(2)}}{4\left(1+i k_{t}\right)} \exp \left(\frac{k_{t} R}{B}\right)\left[\exp \left(-i 2 k_{t} \Delta s_{F}\right)-1\right]\right|
\end{aligned}
$$

\subsubsection{Discrete inverse method}

Harding and Bryden (2012) introduced a discrete inverse method for the solution of the mathematical model. In this method, the wake circulation in Eqs. (13) and (14) is discretized on discrete particles by utilizing the discrete Biot-Savart's law (cf. Eq. (11)). The assumptions (i) and (ii) from Sec. 3.1 are enforced by specifying the particles' position a-priori at the mean chord line, uniformly distributed at distance $\Delta x_{F}$. For the configuration in Fig. 1, the discrete velocities at $\boldsymbol{x}_{c}$ at step $j$ can be obtained as follows:

$$
\begin{aligned}
& u_{c, j}=\sum_{k=j}^{N_{v}+j-1} Z_{u, j k}\left(\Gamma_{F_{A}, k}^{i n}-\Gamma_{F_{B}, k}^{i n}\right), \\
& w_{c, j}=\sum_{k=j}^{N_{v}+j-1} Z_{w, j k}\left(\Gamma_{F_{A}, k}^{i n}+\Gamma_{F_{B}, k}^{i n}\right),
\end{aligned}
$$

where $j=\left\{1, \ldots,\left\lfloor N_{s} / \Delta p\right\rfloor\right\}$ and $N_{v} \in \mathbb{N}$ are number of vortices considered in the spatial range of summation for the inverse model $l_{v}^{i n v}$. The matrices $Z_{u, j k}$ and $Z_{w, j k}$ are obtained by using Eq. (11), considering that at each step $j$, the velocities are influenced only by $N_{v}$ number of vortices. Thus, these matrices are of size $\left\lfloor N_{s} / \Delta p\right\rfloor \times\left\lfloor N_{s} / \Delta p\right\rfloor+N_{v}$, and are obtained as follows:

$$
\begin{aligned}
& Z_{u, j k}=\left\{\begin{array}{cl}
\frac{R}{4 \pi\left[\left(x_{c}-x_{F, k}\right)^{2}+\frac{R^{2}}{4}\right]}, & \text { if } j \leq k \leq j+N_{v}-1, \\
0, & \text { otherwise; }
\end{array}\right. \\
& Z_{w, j k}=\left\{\begin{array}{cl}
-\frac{x_{c}-x_{F, k}}{2 \pi\left[\left(x_{c}-x_{F, k}\right)^{2}+\frac{R^{2}}{4}\right]}, & \text { if } j \leq k \leq j+N_{v}-1, \\
0, & \text { otherwise. }
\end{array}\right.
\end{aligned}
$$

Equations (27) can be reversed and the circulation can be obtained for a prescribed velocities, $u_{c t}$ and $w_{c t}$, as follows:

$$
\left\{\begin{array}{l}
\Gamma_{F_{A}, k}^{i n}-\Gamma_{F_{B}, k}^{i n}=\sum_{j=1}^{N_{s}} Z_{u, k j}^{+} u_{c t, j} \\
\Gamma_{F_{A}, k}^{i n}+\Gamma_{F_{B}, k}^{i n}=\sum_{j=1}^{N_{s}} Z_{w, k j}^{+} w_{c t, j}
\end{array}\right.
$$

where $Z_{u, k j}^{+}$and $Z_{w, k j}^{+}$are the pseudo-inverse matrices of $Z_{u, j k}$ and $Z_{w, j k}$, respectively, as no unique inverse is feasible since the system is overdetermined. The Moore-Penrose procedure is utilized for the pseudo-inverse operation. Equations (29) are solved as simultaneous equations at each step. Harding and Bryden (2012) further relate the circulation $\Gamma_{F}^{i n}$ to the airfoil angles $\alpha_{F}$.

The inverse method is given herein to verify the closed-form solution derived in Eq. (26). Furthermore, it is noted that the prescribed velocities in the inverse method by Eq. (29) do not need to be sinusoidal. In fact, they can be arbitrary functions, as experimentally validated by Harding et al. (2014). Thus, the inverse method is more flexible than the analytical; however, the latter is preferred for sinusoidal functions as it is more computationally efficient and avoids the error in least-squares fit in the pseudo-inverse operation. This error in the inverse method for sinusoidal velocity fluctuations can be reduced, up to a certain extent, by taking longer $l_{v}^{i n v}$ than the spatial length of summation $l_{v}$ of the CFD domain (cf. Fig. 1). 


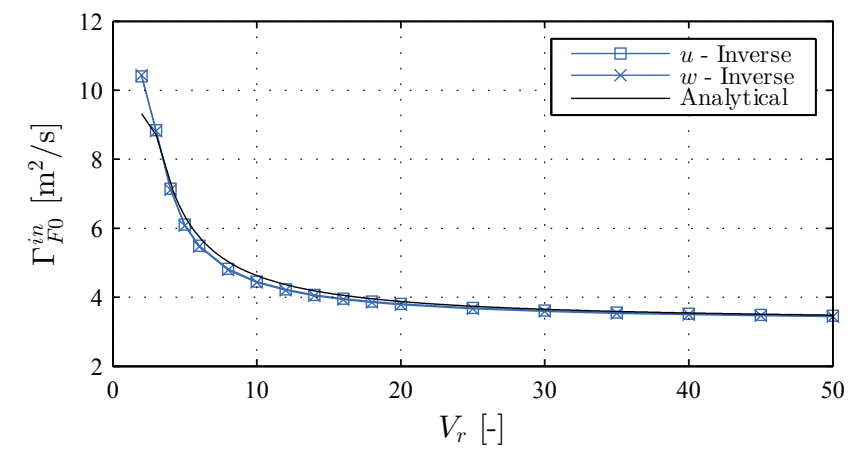

Figure 2: Verification of the analytical closed-form solution (cf. Eq. (26)) with the inverse method (cf. Eq. (29)) for circulation amplitude prediction of the released particles $\Gamma_{F 0}^{i n}$.

\section{Verification}

In this section, we start by investigating the gust amplitude and quality in the flow field from CFD simulations without a section. Further, we verify the CFD aerodynamic admittance for a flat plate with Sears' admittance function. Finally, the aerodynamic admittance for deterministic and random freestream turbulence are compared.

\subsection{Flow field}

To study the flow field for the presented numerical ATG we define a basic configuration using the following parameters: domain length $l_{d} / B=21$, vertical airfoil distance $R / B=1$, Reynolds number $\operatorname{Re}=U B / v=1 \times 10^{4}$, and reduced simulation time-step $\Delta s=5 \times 10^{-3}$. The particle release factor $\Delta p$ is selected as such to accommodate either 30 particles per gust length or a minimum of $N_{v}=200$ particles for the spatial range of summation $l_{v}$. With this condition, the particle release factor amounts to $12 \leq \Delta p \leq 21$; hence, the reduced time-step of the released particles is in the range of $6 \times 10^{-2} \leq \Delta s_{F} \leq 10.5 \times 10^{-2}$ for the prescribed reduced velocity range of $2 \leq V_{r t} \leq 50$. Two cases are studied, in which the fictitious airfoils are assumed to be oscillating out-of- and in-phase, generating longitudinal and vertical sinusoidal gusts, respectively. In the following, we recall these two cases as the "out-of-phase" and the "in-phase" case. For both cases, a similar gust amplitude along the centerline is prescribed, that is, $u_{c t 0} / U=w_{c t 0} / U=0.05$.

Initially, the given closed-form solution for the analytical method (cf. Eq. (26)) for the computation of the circulation of the amplitude of the released particles $\Gamma_{F 0}^{\text {in }}$ is verified with the inverse one (cf. Eqs. (29)). Figure 2 depicts the circulation obtained for the selected reduced velocity range and oscillation amplitudes. The circulation amplitude corresponds well for both methods in the prescribed reduced velocity range, except at $V_{r t}=2$, where issues were noted in the pseudo-inverse procedure for the inverse method.

Figure 3 depicts time-histories of the velocity fluctuations and their corresponding FFTs at point $\boldsymbol{x}_{c}=(5 \mathrm{~B}, 0)$ at $V_{r t}=16$ for both cases. A clear sinusoidal signals can be observed for the longitudinal and vertical velocity fluctuations. In the inphase case, there is a longitudinal component in addition to the vertical fluctuations. However, the amplitude of this component is an order of magnitude lower than the amplitude of the vertical fluctuations. Theoretically, for in-phase motion of the airfoils, there should not be a longitudinal component. Moreover, the vertical fluctuation amplitude is slightly underestimated.

To study the origin of these discrepancies, Fig. 4 presents instantaneous particle maps for three reduced velocities and an instantaneous velocity vector field at $V_{r t}=16$, for both cases. It can be observed that the particles are not convected along a horizontal line, particularly for the in-phase case. The planar-wake assumption is more reasonable for the out-of-phase case, as the instability of one wake is counteracted by the other. On the contrary, this instability is enhanced in the in-phase case. Thus, the discrepancies in the gust amplitudes and the secondary fluctuating components are a consequence of violating the planar wake and non-interference assumptions, noted in Sec. 3.1. As expected, this discrepancy is more prominent for lower reduced velocities in the neighborhood of the particle release locations due to the variation of the circulation over shorter gust-lengths. The sinusoidal variation of the velocity for the out-of-phase case is only between the wakes, while for the in-phase case there is variation outside this region (cf. Fig. 4, bottom). Nevertheless, it is important that the body is position within the region between the two wakes to avoid the absorption of particles entering the body.

For the computation of the aerodynamic admittance it is important that the body is immersed in a uniform sinusoidal gust, while the gust amplitude is of minor significance as the aerodynamic forces are considered to be linear. In order to correctly position a section within the domain, it is of interest to study the gust uniformity and its harmonic component. Hence, we introduce a relative quality parameter $Q$. This parameter quantifies the energy of a fluctuating velocity at a single prescribed frequency $f_{t}$ relative to the total energy of both fluctuating velocities. The gust quality for the out-of-phase and in-phase cases, respectively, is defined as follows:

$$
Q_{u}=\frac{S_{u}\left(f_{t}\right)}{\int_{0}^{\infty}\left[S_{u}(f)+S_{w}(f)\right] \mathrm{d} f}, Q_{w}=\frac{S_{w}\left(f_{t}\right)}{\int_{0}^{\infty}\left[S_{u}(f)+S_{w}(f)\right] \mathrm{d} f},
$$

where $S_{u}=S_{u}(\boldsymbol{x}, f)$ and $S_{w}=S_{w}(\boldsymbol{x}, f)$ are the PSDs of the longitudinal and vertical fluctuations, respectively, at location $\boldsymbol{x}$. Therefore, the gust quality $Q$ and relative gust amplitude ratios, $u_{0} / u_{t 0}$ and $w_{0} / w_{t 0}$, are the quantities of interest of the flow field for the purpose in this work.

Figure 5 depicts the influence of the reduced velocity on flow quantities of interest along the center line $\boldsymbol{x}_{c}=\left(x_{c}, 0\right)$ for both cases. After the particle release location, there is a certain length, in which the gust amplitudes converge (cf. Fig. 5, left). This convergence length is longer for the in-phase case and is also dependent on the reduced velocity. In the out-ofphase case, the gust amplitudes generally agree well with the prescribed ones. In the in-phase case the situation is slightly different. Beyond the convergence length, the gust amplitudes begin to "wobble", which is probably due to the distortion of the particle path (cf. Fig. 4, top-right). For gust lengths which are longer than half of the domain (i.e. $V_{r t} \geq 10$ ), the conver- 

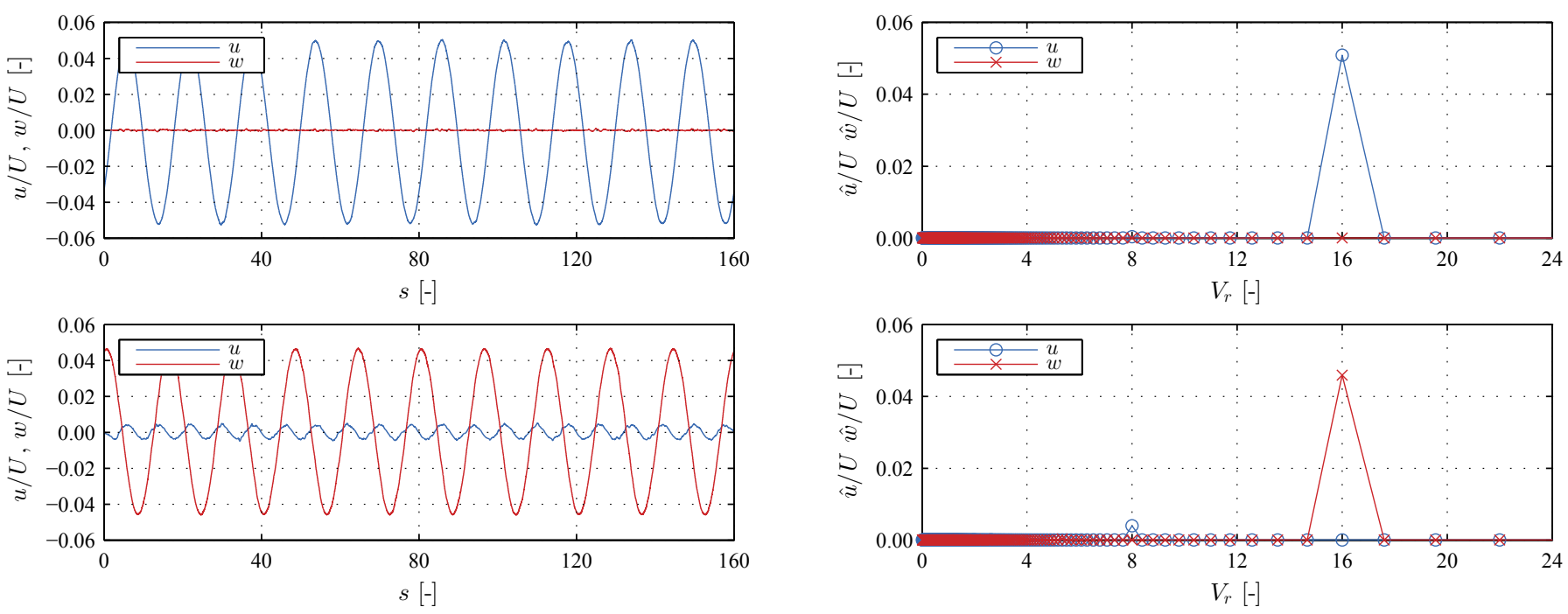

Figure 3: Time-histories of velocity fluctuations (left) and their corresponding FFTs (right) for airfoils oscillating out-of-phase (top) and in-phase (bottom). The gust tracking point is $\boldsymbol{x}_{c}=(5 B, 0)$ at $V_{r t}=16$.
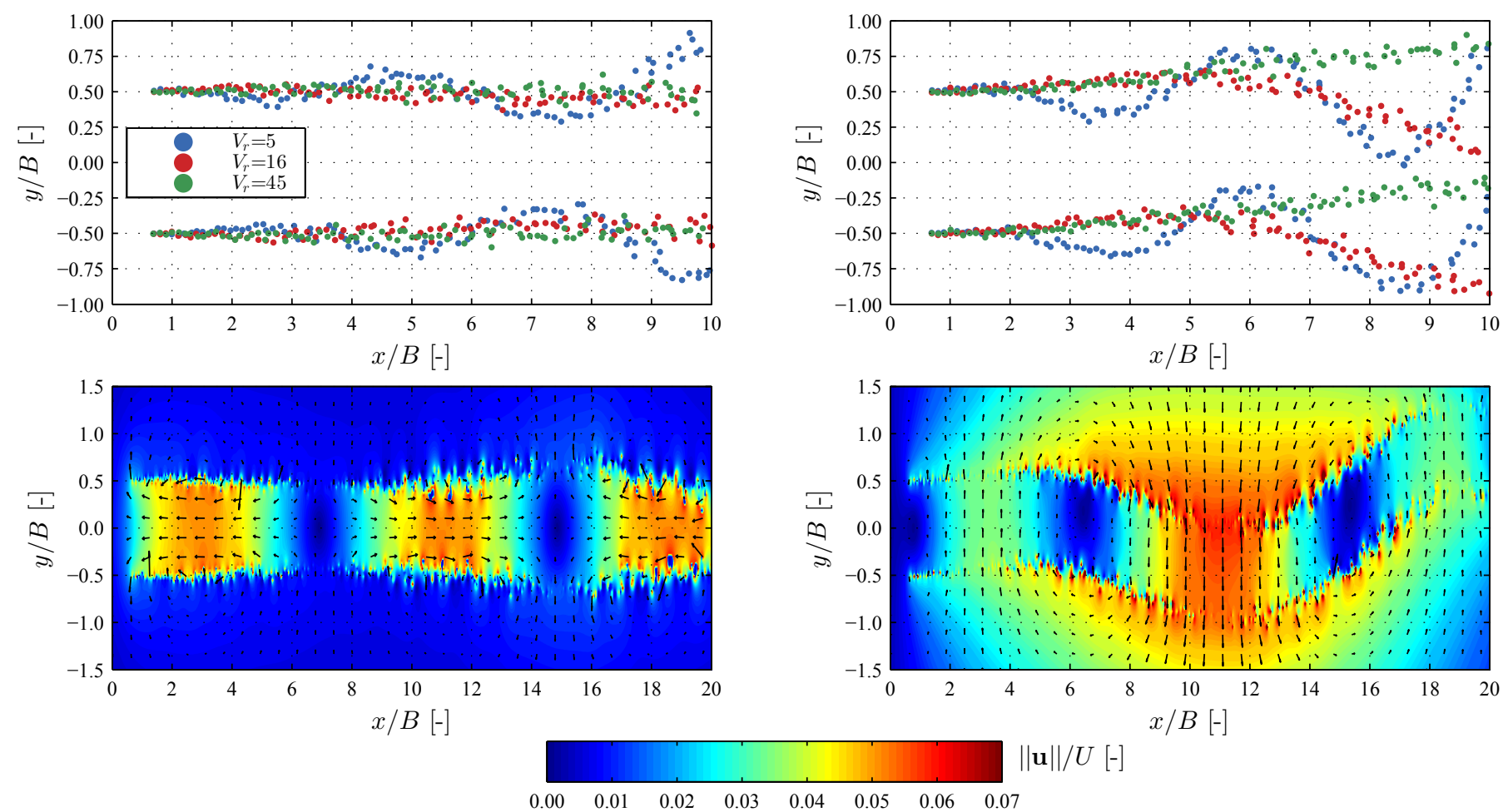

Figure 4: Instantaneous particle maps (top) and instantaneous velocity vector fields at $V_{r t}=16$ (bottom) for airfoils oscillating out-of-phase (left) and in-phase (right).

gence length is not reached and this behavior is not observed. The prediction of the magnitude of oscillation is within the $\pm 20 \%$ range, taking the "wobbling" into account in the region of $2<x_{c} / B<10$. For the highest reduced velocity $V_{r t}=45$, it can be observed that gust amplitude reached up to $80 \%$ of the prescribed one, i.e. $w_{c 0} / w_{c t 0}=0.8$.

As noted previously, the gust quality $Q$ is more important than the agreement of the gust amplitude with its prescribed value for the aerodynamic admittance. Figure 5 depicts the quality parameters for the out-of-phase (top-right) and in-phase (bottom-right) case. It can be observed that the quality decays for points which are further downstream of the particle release locations for the in-phase case. This decay is faster for the lower reduced velocities, due to faster distortion of the path of released particles w.r.t. the prescribed horizontal path. Nevertheless, for a quality parameter more than 0.9 , it can be assumed 

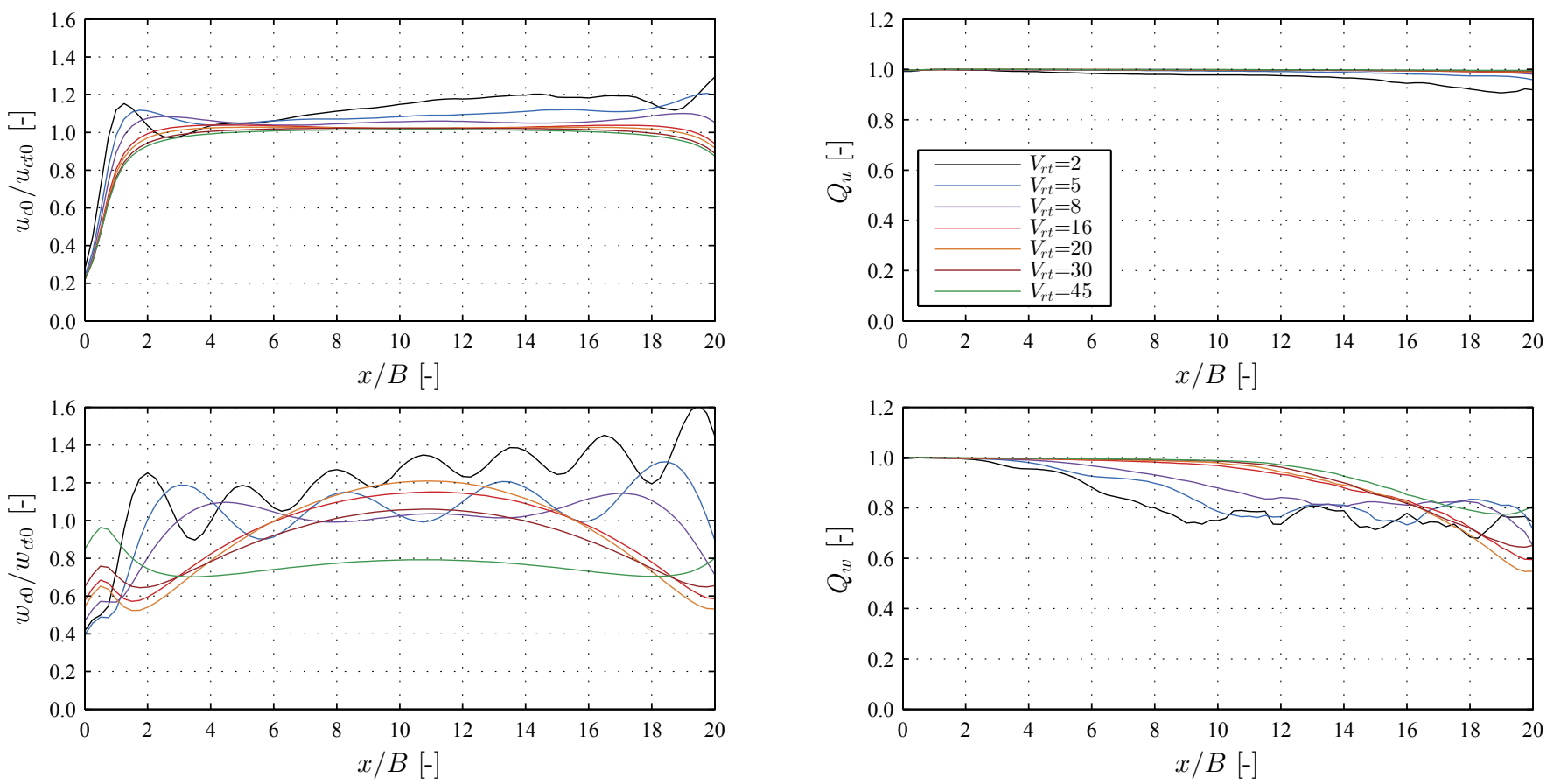

Figure 5: Influence of the reduced velocity on the relative gust amplitude (left) and quality (right) for airfoils oscillating out-of-phase (top) and in-phase (bottom) along the centerline $y=0$.
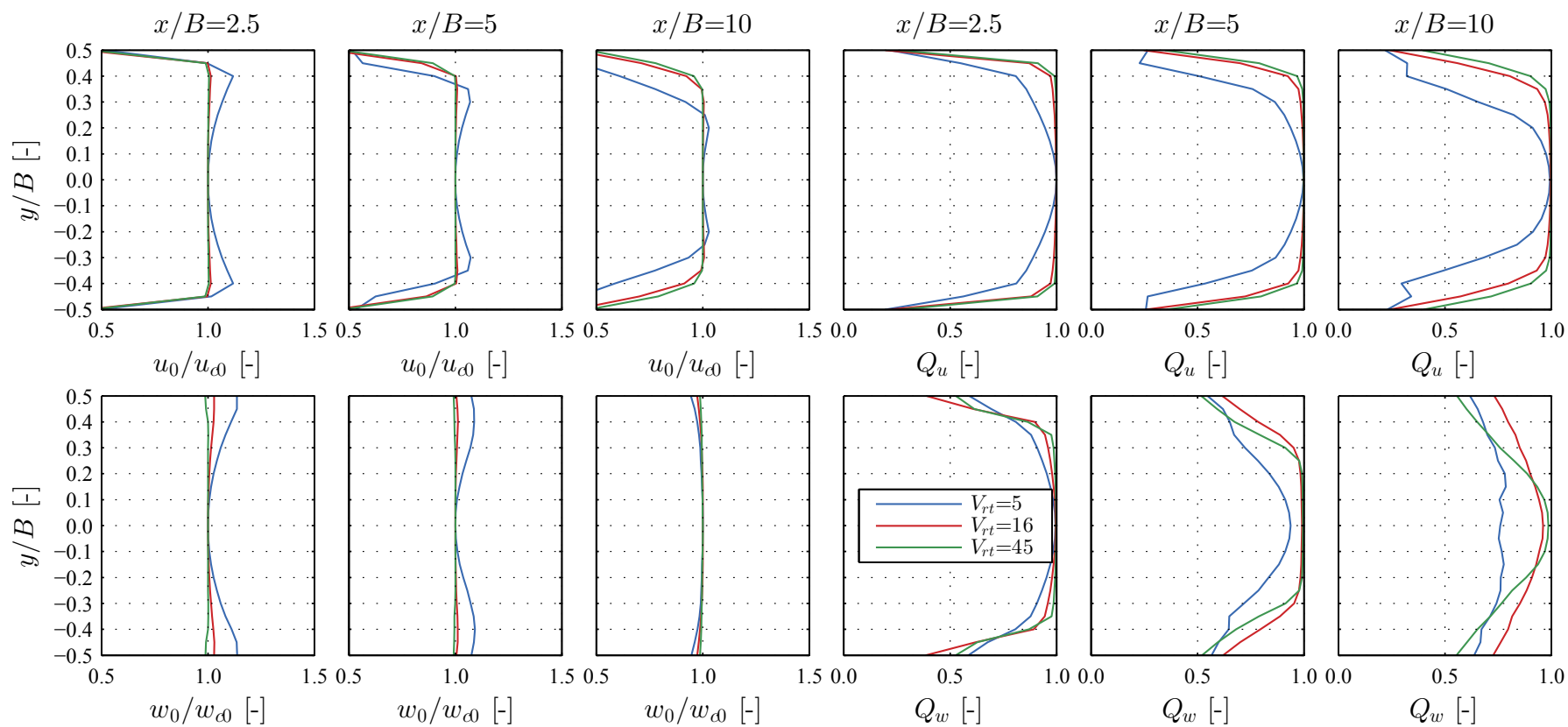

$Q_{u}[-]$

Figure 6: Influence of the reduced velocity on the relative gust amplitude (left) and quality (right) between the particle release locations for airfoils oscillating out-of-phase (top) and in-phase (bottom).

that the gust is considered as sinusoidal and most of the fluctuating energy is concentrated at a single vertical frequency. For the out-of-phase case, the gust quality along the centerline is mostly above 0.9 .

Besides sufficient gust quality and amplitude uniformity along the centerline, it should be ensured that these quantities are uniform along the depth of the section. Figure 6 depicts the deviation of the gust amplitude along the height w.r.t. the one at the center line for three vertical profiles. Moreover, the gust quality is included in the figure. The difference in the amplitude is appearing mostly for the out-of-phase case nearby the particle release locations and is more prominent for low reduced velocities. This is in line with the observations noted previously based on the velocity vector field (cf. Fig. 4, bottom-left). For the 
in-phase case, the deviation of the gust amplitude is even less obvious. Generally, the deviation of the gust amplitude along the height is relatively mild for both cases. The discrepancies of gust quality along the height are somewhat larger than the gust amplitudes. It can be observed that there is certain band w.r.t. the center line, for which the quality is relatively constant (cf. Fig. 6, right). This band reduces for vertical profiles, positioned further downstream of the particle release locations. It is important that the section is within the limits of this band when the aerodynamic admittance is computed.

Stapountzis (1982) introduces a non-dimensional gust intensity to quantify the relation between the gust amplitude and oscillation amplitude of the airfoils. The ratio for the longitudinal and vertical gust amplitudes, respectively, is given as follows:

$$
I_{g u}=\frac{u_{c 0}}{U \alpha_{F 0}}, \quad I_{g w}=\frac{w_{c 0}}{U \alpha_{F 0}} .
$$

In case of the analytical method for the solution of the mathematical model, this relation is in closed-form. For the inverse method, the input variable is the gust intensity, $u_{c t 0}$ or $w_{c t 0}$, while $\alpha_{F 0}$ is obtained from the circulation (cf. Eqs. (29)) (Harding and Bryden, 2012). The input variable in the CFD model is the airfoil amplitude $\alpha_{F 0}$, since it is directly related to the circulation amplitude $\Gamma_{F 0}^{i n}$. while the tracked gust intensity $u_{c 0}$ or $w_{c 0}$ is the output variable. Figure 7 depicts the non-dimensional gust intensities for the CFD and mathematical model, the latter solved using the inverse and analytical methods. For the CFD model, the point for the gust amplitude is the selected location of the stiffness center of the section is positioned, which is specified in the following section. Generally, good correspondence is obtained for the models, with small discrepancies for very low reduced velocities and for gust lengths which are significantly longer than the domain length for the in-phase case.

Parametric studies are conducted by varying the domain length $l_{d}$, gust amplitudes $u_{c t 0}$ and $w_{c t 0}$, and airfoil distance $R$. For the sake of brevity, the results are given in Appendix A (cf. Figs. A.24-A.26). It is observed that: (i) the vertical gust amplitudes for high reduced velocities are in better correspondence with the prescribed values for increasing $l_{d} / B$ ratio; (ii) the quality of the vertical gusts decays more rapidly for higher gust amplitudes; and, (iii) increasing the $R / B$ ratio increases the vertical gust quality for high reduced velocities. However, for

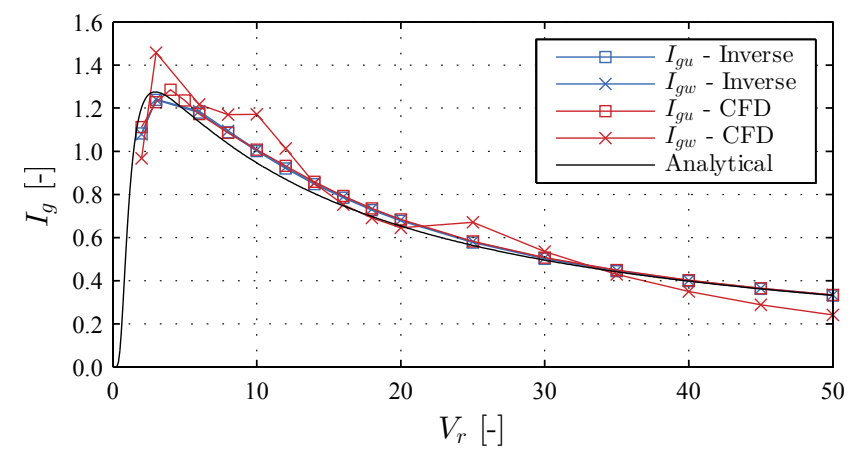

Figure 7: Dimensionless gust intensity for the inverse and analytical solution of the mathematical model and CFD model. very large $R / B$ ratios, the quality of the gust is expected to be reduced. The reason for this are the high circulation amplitudes required for a specific gust amplitude, which would effectively contribute to the violation the planar wake assumption.

\subsection{Flat plate aerodynamic admittance}

The aerodynamic admittance is verified for a flat plate with width-to-depth ratio of 200 with the analytical Sears' admittance. For the verification, the complex form of Sears' function is used, given as follows (Sears, 1941):

$$
\chi=F+i G=\left(J_{0}-i J_{1}\right) C+i J_{1}
$$

where $C=C(k)$ is the Theodorsen's function (cf. Eq. (21)), while $J_{0}=J_{0}(k)$ and $J_{1}=J_{1}(k)$ are Bessel functions of the first kind.

Following the flow field verification in the previous section, the stiffness center $\boldsymbol{x}_{s}=\left(x_{s}, 0\right)$ of the flat plate is positioned at $x_{s} / B=2$ for $V_{r t} \leq 4 ; x_{s} / B=5.5$ for $4<V_{r t}<16$; and $x_{s} / B=10$ for $V_{r t} \geq 16$. The flow parameters and the domain are similar as in the previous section for the in-phase case. With this, a gust quality above $Q \geq 0.9$ is ensured the section region and the released particles are not crossing the body. The section is discretized on 400 panels. Again, the Reynolds number is set as $\operatorname{Re}=U B / v=1 \times 10^{4}$, for which the boundary layer is not broken and the viscous effects are negligible.

The analytical aerodynamic admittance, given by Sears (1941), is based on the linear assumption and potential flow. Hence, the boundary layer and the static wind coefficients are verified for smooth free-stream before subjecting the flat plate to sinusoidal gusts. Figure 8 (left) depicts the boundary layer w.r.t. the similarity parameter, that is $\eta=y \sqrt{U /(v x)}$, for the top and bottom surface at three locations along the flat plate $x \in\left\{x_{s}-B / 4, x_{s}, x_{s}+B / 4\right\}$. Good correspondence can be observed w.r.t. the analytical solution given by Blasius (1950), and the symmetry of the flow is preserved. The lift and moment static wind coefficients w.r.t. the static angle of attack $\alpha_{s}$ are given in Fig. 8 (right). These values are further compared to their analytical counterparts, computed for lift and moment slopes of $C_{L}^{\prime}=2 \pi$ and $C_{M}^{\prime}=0.5 \pi$, respectively. From the CFD analyses, the lift and moment slopes amount to $C_{L}^{\prime}=1.94 \pi$ and $C_{M}^{\prime}=0.46 \pi$, resulting in $3.8 \%$ and $7.3 \%$ difference, respectively, w.r.t. analytical counterparts.

\subsubsection{Deterministic free-stream turbulence}

Next, the flat plate is subjected to vertical sinusoidal gusts with reduced velocities in the range of $2 \leq V_{r t} \leq 50$. Figure 9 depicts a sequence of particle maps from a cycle of sinusoidal gust past a flat plate at prescribed reduced velocity of $V_{r t}=16$. It can be seen that even at the peak velocities ( $s=17.5$ and $s=25.5)$, the released particles do cross the body. To obtain the aerodynamic admittance, the sinusoidal gust needs to be tracked down at a specific location $\boldsymbol{x}_{c}$ within the CFD domain to obtain the actual gust amplitude. For this case, the gust is tracked at the stiffness center, that is $\boldsymbol{x}_{c} \equiv \boldsymbol{x}_{s}$, as described in the optional step c) from Sec. 3.1. Hence, the influence of the body on the upstream 

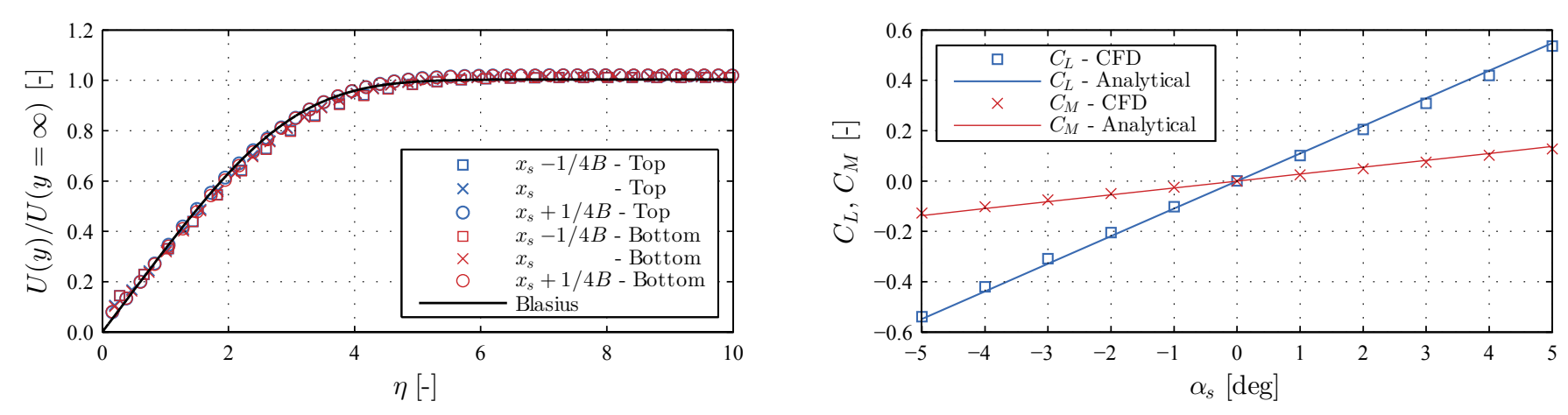

Figure 8: Boundary layer (left) and static wind coefficients (right) for a flat plate.
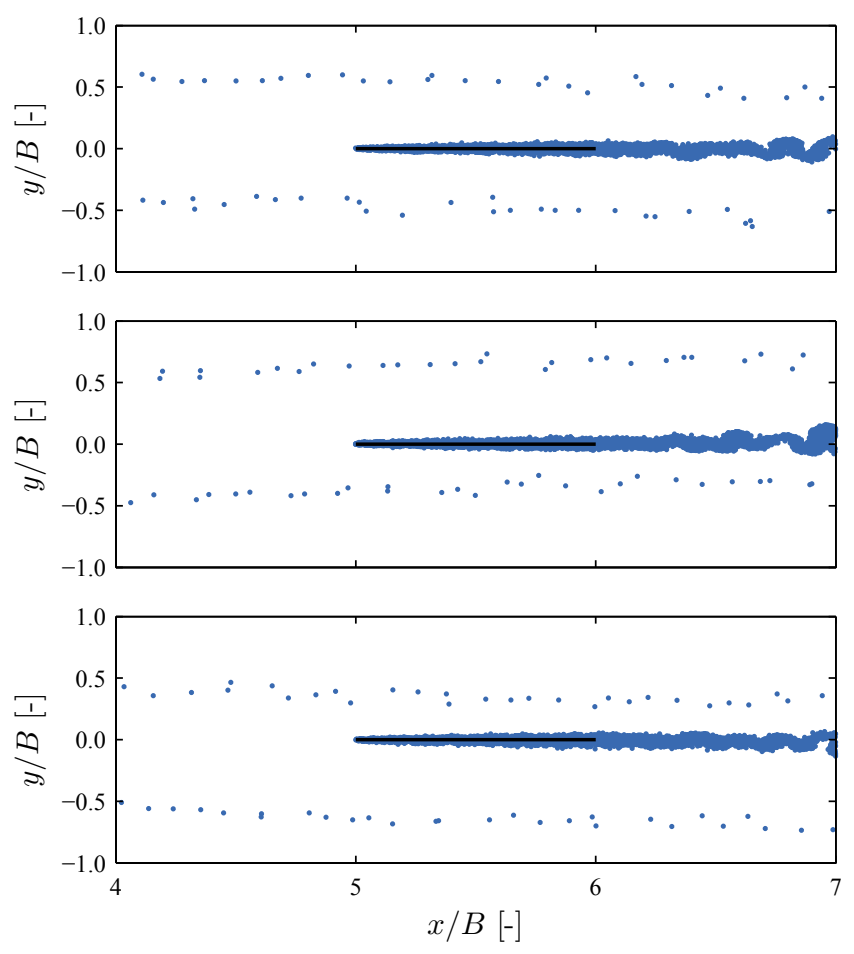

Figure 9: Sequence of instantaneous particle maps for a sinusoidal vertical gust past a flat plate at prescribed reduced velocity $V_{r t}=16: s=13.5$ (top); $s=17.5$ (center); $s=25.5$ (bottom).

velocity is not taken into account. Nevertheless, the linear unsteady model (cf. Eqs. (4)), based on the potential flow theory, does not account for this effect in anyway. Moreover, the tracking location can be positioned arbitrarily far upstream of the leading edge. This leads to inconsistent results as the influence of the section depends on the distance between the tracking location and leading edge. Tracking the gust at the stiffness center $\boldsymbol{x}_{s}$ is only applicable for CFD analyses, as the simulations with and without section are reproducible. In experiments, the gust needs to be tracked down at a point upstream of the leading edge, as seen in Sec. 5.

Figure 10 (left) depicts a time-history of the lift and moment fluctuating coefficients at $V_{r t}=16$ normalized w.r.t. gust angle. These are further compared to their analytical counterparts for the Sears' function. From the figure, it can be observed that the forces sinusoidal. The additional high frequency fluctuations for the CFD model are caused by the viscous random walk and vortex-shedding. It could be observed that Sears' fluctuating coefficient is sort of a least-square fit to the results from the CFD analyses. The FFTs of the normalized coefficients (cf. Fig. 10, right) also depicts a clear harmonic in the forces.

Figure 11 (left) depicts the real $F$ and imaginary $G$ part$\mathrm{s}$ of the complex aerodynamic admittance obtained from the CFD simulations and the Sears' function. Moreover, the absolute value of the aerodynamic admittance (cf. Fig. 11, right) is given. The aerodynamic admittance is computed based on 10 cycles of sinusoidal gust for each reduced velocity. Not only there is a good agreement for the absolute values with the Sears' function, but also the separate real and imaginary part correspond well. As it can be observed from the figure, the gust lags behind the aerodynamic forces for reduced velocities up to $V_{r t}=8$. Another important particularity is that the aerodynamic admittance of the moment and lift force coincide, which effectively means that the lift force is acting on the front quarterchord point. A maximum deviation of $10 \%$ for the absolute value of the admittance is obtained for a prescribed reduced velocity of $V_{r t}=2$, while the discrepancies are in the range of $\pm 2 \%$ for higher reduced velocities $V_{r t}>3$.

\subsubsection{Random free-stream turbulence: Comparison}

The results obtained in the previous section are further compared with the results for the method with random free-stream turbulence by Rasmussen et al. (2010).

The method consists of releasing particles from a "band" of release locations. This band represents multiple particle release locations, positioned on a line upstream of the section, perpendicular to the mean wind. The circulation of the particles are obtained from a velocity field with prescribed spectral characteristics. Releasing the particles upstream simulates a random velocity field within the domain. Herein, we do not describe the method in detail for the sake of brevity, rather give the required numerical parameters. For further information on the method, we refer the reader to Kavrakov and Morgenthal (2018a,b); Prendergast (2007).

For the generation of the velocity field, herein the von Kármán PSD function is utilized as it yields better correspondence 

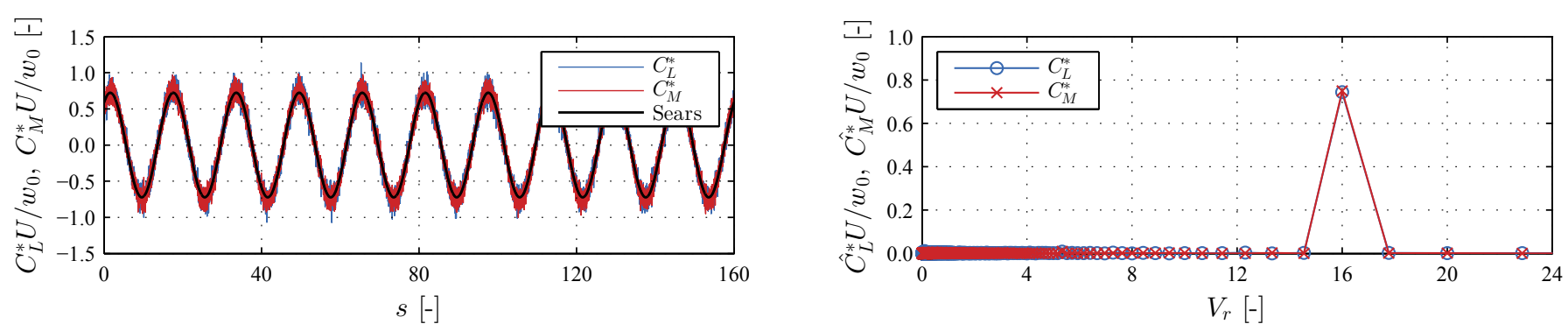

Figure 10: Normalized fluctuating wind coefficients (left) and their corresponding FFTs for a flat plate at prescribed reduced velocity $V_{r t}=16$.
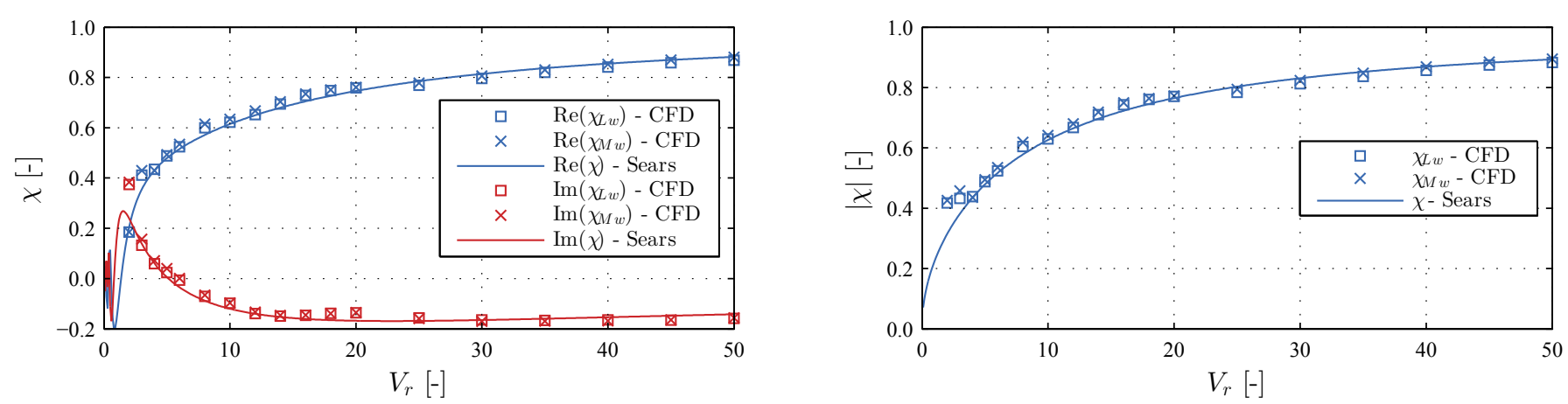

Figure 11: Verification of the aerodynamic admittance for a flat plate: real and imaginary part (left); absolute value (right).

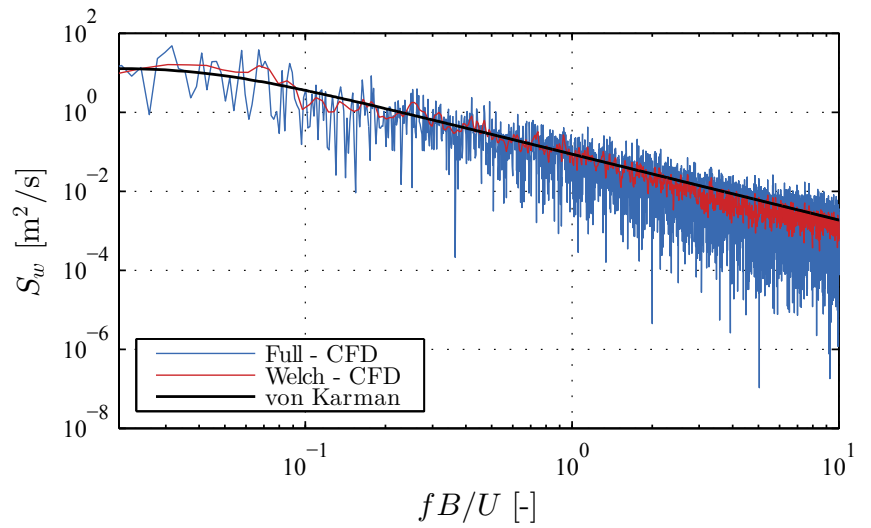

Figure 12: PSD of the vertical fluctuating component for random free-stream turbulence at the gust tracking point $\boldsymbol{x}_{c}=$ $(10.5 B, 0)$.

(Kavrakov and Morgenthal, 2018b) for isotropic turbulence than the ESDU PSD, which was used by Rasmussen et al. (2010). The von Kármán PSD is given as follows (Solari and Piccardo, 2000):

$$
\begin{aligned}
\frac{f S_{u}}{\sigma_{u}^{2}} & =\frac{4 f L_{u} / U}{\left[1+70.8\left(f L_{u} / U\right)^{2}\right]^{5 / 6}}, \\
\frac{f S_{w}}{\sigma_{w}^{2}} & =\frac{4 f L_{w} / U\left[1+755.2\left(f L_{w} / U\right)^{2}\right]}{\left[1+283.2\left(f L_{w} / U\right)^{2}\right]^{11 / 6}},
\end{aligned}
$$

where $\sigma_{u}$ and $\sigma_{w}$ is the standard deviation of the longitudinal and vertical fluctuations, respectively, while $L_{u}$ and $L_{w}$ are the longitudinal and vertical length-scales. For the analyses, the turbulence intensity amounts to $\sigma_{u} / U=\sigma_{w} / U=0.05$, while the length-scales are set as $L_{u}=4 B$ and $L_{w}=2 B$. Vickery's coherence function is utilized with horizontal and vertical coherence coefficients amounting to 3 and 10, respectively (Solari and Piccardo, 2000). The height of the band is set as $16 B$ and positioned $10 B$ upstream of the leading edge. The particle release factor amounts to $\Delta p=4$, resulting in 800 particle release locations. The flow field in the CFD analyses results in very good correspondence with the isotropic von Kármán PSD (Kavrakov and Morgenthal, 2018b). As an example, the PSD of the vertical component for the considered reduced velocity range is given in Fig. 12 at gust tracking point $\boldsymbol{x}_{s} \equiv \boldsymbol{x}_{c}=(10.5 B, 0)$ within the CFD domain for a simulation without a section.

When random free-stream fluctuations are considered as "input", the determination of the aerodynamic admittance is obtained for the whole reduced velocity range. Hence, the aerodynamic admittance for the lift and moment from Eqs. (4) yields the following:

$$
\begin{aligned}
\left|\chi_{L}\right|^{2} & =\frac{4 S_{L_{b}}}{\rho^{2} U^{2} B^{2}\left[4 C_{L}^{2} S_{u}+\left(C_{L}^{\prime}+C_{D}\right)^{2} S_{w}\right]}, \\
\left.\chi_{M}\right|^{2} & =\frac{4 S_{M_{b}}}{\rho^{2} U^{2} B^{4}\left(4 C_{M}^{2} S_{u}+C_{M}^{\prime}{ }^{2} S_{w}\right)},
\end{aligned}
$$

where $S_{L_{b}}$ and $S_{M_{b}}$ are the PSDs of the lift and moment buffeting forces, respectively.

Figure 13 depicts the absolute value of the aerodynamic admittance for a flat plate obtained using the sinusoidal and ran- 

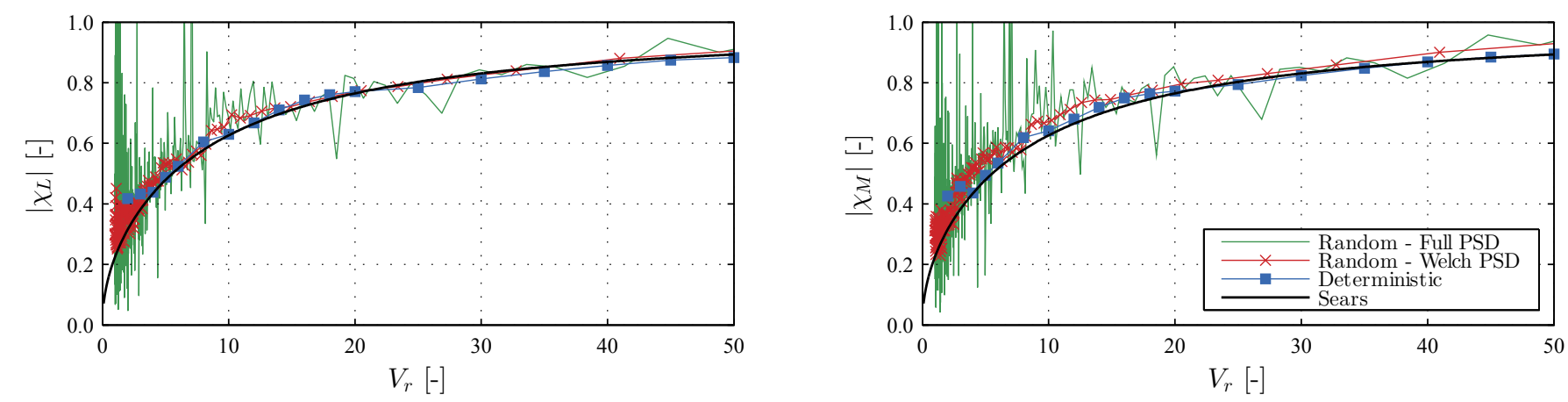

Figure 13: Comparison of lift (left) and moment (right) aerodynamic admittance of a flat plate for deterministic and random freestream turbulence.

dom free-stream fluctuations. The aerodynamic admittance obtained using random free-stream fluctuations is computed by dividing two PSDs (cf. Eqs. (34)). Significant numerical noise is noted for the aerodynamic admittance if the full PSDs are used, without any smoothing (cf. Fig. 13). This numerical noise can be reduced if PSDs are obtained using the Welch's method.

From the results, it can be observed that the results from both sinusoidal and random free-stream (using Welch's PSD) correspond well with Sears' admittance, with slightly better agreement for the moment admittance from deterministic freestream turbulence. Some advantages of utilizing deterministic as opposed to random free-stream turbulence include the following: (i) the admittance is obtained in its complex for$m$ rather than only the absolute value, (ii) all six components of the aerodynamic admittance can be obtained separately (cf. Eqs. (5)) instead of only three, and, (iii) the numerical uncertainty arising from the smoothing in the PSD is avoided. As a consequence of the latter point, it is difficult to separate the contribution of the incident part from the shear layer and wake fluctuations of the admittance for the random free-stream. A drawback of obtaining the admittance using sinusoidal gusts is the computational inefficiency, since a separate CFD simulation should be performed for each reduced velocity.

It is important to note that the analogy between deterministic and random free-stream turbulence for the 2D VPM is not the same as the analogy in experiments between gusts obtained using an ATG and grid turbulence. The 3D effects in the admittance obtained using random free-stream turbulence can be dominant in experiments, while the random free-stream turbulence in the VPM remains 2D. Hence, the absolute values of the CFD aerodynamic admittance for both methods are comparable.

\section{Validation}

In this section, we validate the presented method with experimental results from wind tunnel tests for a streamlined deck. Initially, we describe the experimental setup, followed by validation of the static wind coefficients and aerodynamic admittance for vertical sinusoidal gusts. Moreover, the aerodynamic admittance for longitudinal gusts is obtained from CFD sim- ulations. Finally, the CFD lift and moment aerodynamic admittances based on deterministic gusts are compared with their counterparts based on random free-stream turbulence.

\subsection{Experimental setup}

The streamlined deck of the $3^{\text {rd }}$ Bosphorus bridge (also known as the Yavuz Sultan Selim bridge) is selected as a deck for the validation of the CFD model. Static wind coefficients and aerodynamic admittance were measured at the Politecnico di Milano boundary layer wind tunnel using a rigid sectional model from carbon fiber. The model has a geometrical scale of 1:50, with deck width of $1.17 \mathrm{~m}$, depth of $0.11 \mathrm{~m}$, and length of 3.6 m (cf. Fig.14).

The aerodynamic forces were obtained integrating the pressure measured by pressure taps, connected to high frequency pressure scanners. In total, four rings of pressure taps were positioned along the length of the deck to measure the pressure correlation in the axial direction, each ring consisting of 62 taps (cf. Fig.14). The incoming wind measurement is performed one chord before the leading edge by means of a 4-holes probe that resolves the instantaneous vertical and horizontal wind components. Each ring of pressure taps corresponds to a multi-hole Cobra probe. The bridge was tested in a simplified and naked configuration without wind-shields, only including fences. The aerodynamic forces acting on the fences are assumed to be negligible w.r.t. aerodynamic forces acting on the deck; however, they might influence the local aerodynamic properties as discussed further. Thus, the aerodynamic forces are obtained directly through the integration of the pressure distribution from the pressure taps on the deck only. Sectional model tests conducted in such manner implicitly consider that the deck aerodynamics is two-dimensional; hence, the strip assumption is valid.

Sinusoidal gusts were generated by an ATG, constituted of ten airfoils NACA 0012 with chord of $20 \mathrm{~cm}$, positioned at distance of $6 B$ upstream of the section. The ATG is $4 \mathrm{~m}$ wide, driven by two brushless motors giving a pitching motion with a user-defined law in terms of frequency contents and amplitude. An overall picture of the experimental setup in wind tunnel is given in Fig. 14.

The wind tunnel residual turbulence intensity in the horizontal and vertical direction amounted to $\sigma_{u} / U=1.6 \%$ and 
a)

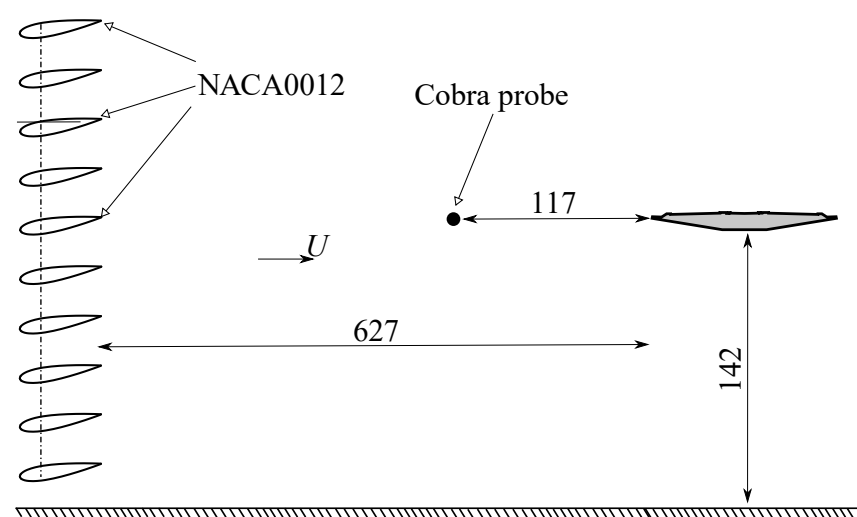

d)

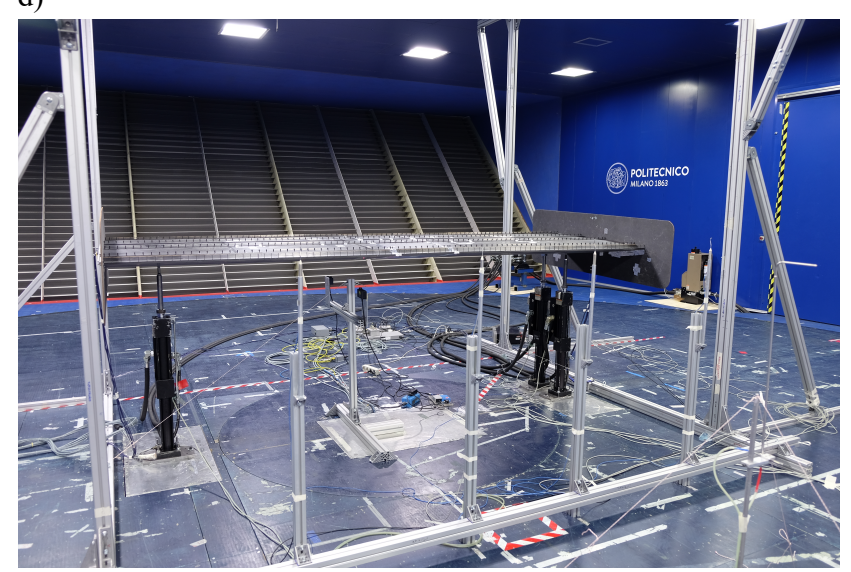

b)

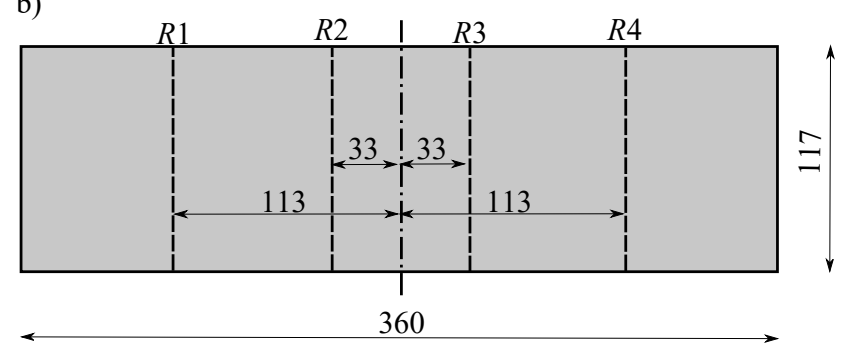

c)

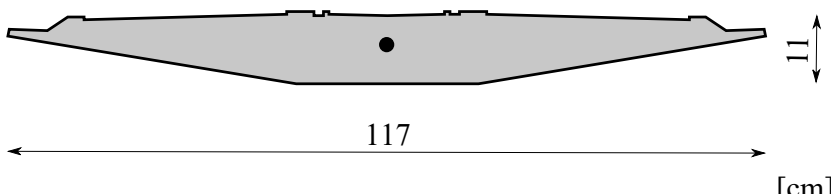

e)
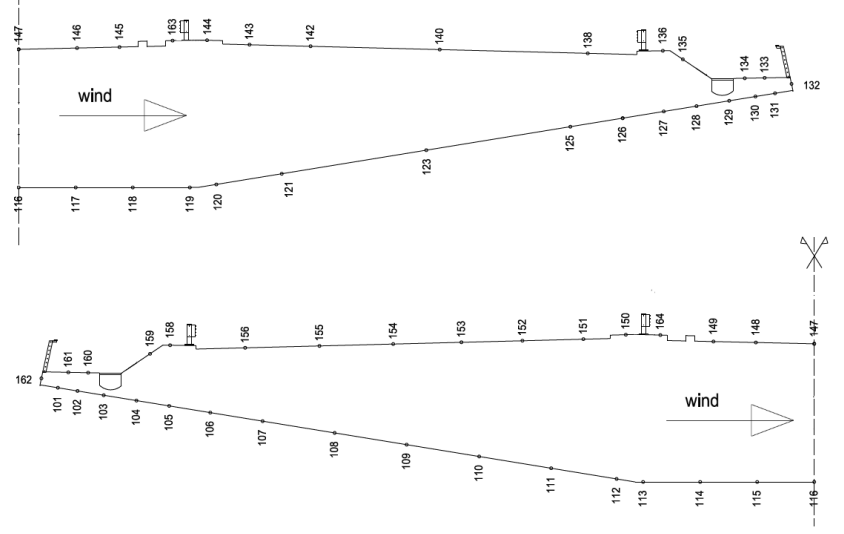

f)

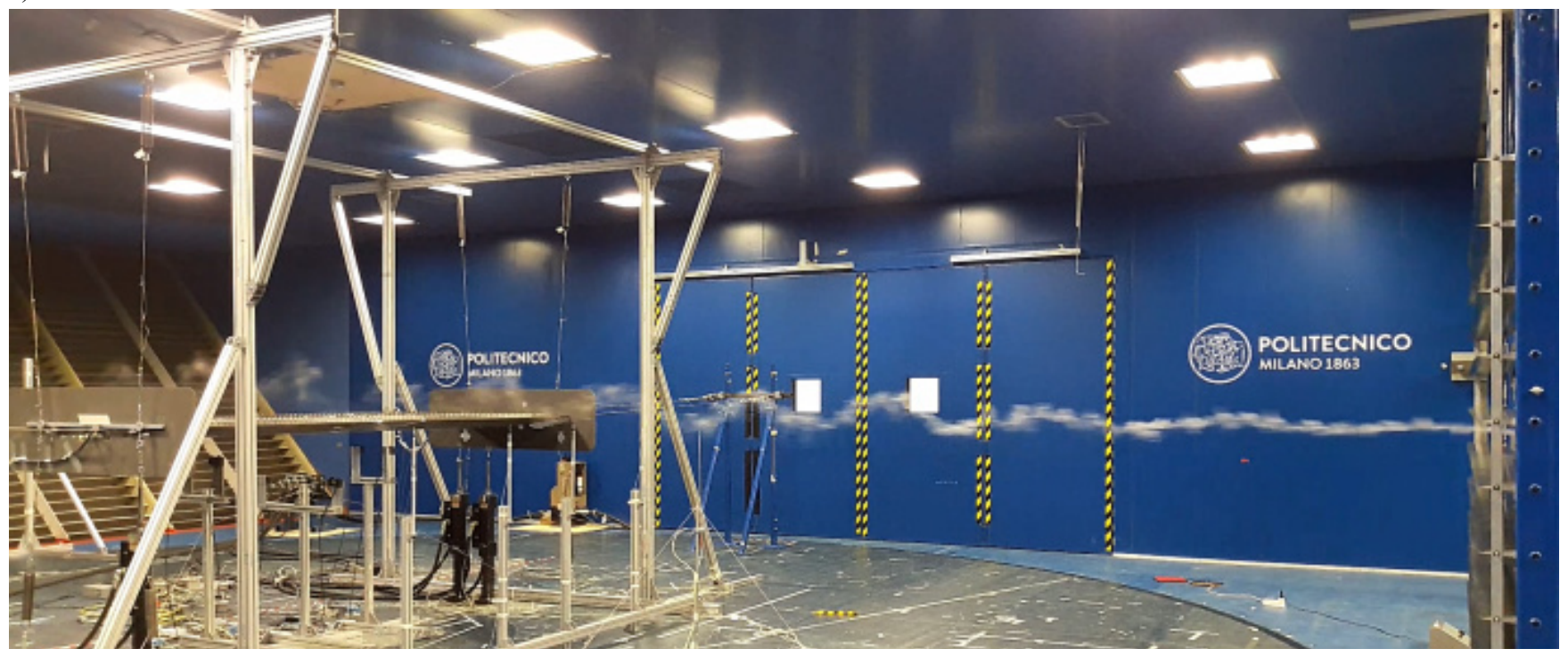

Figure 14: Experiments: a) setup, b) plan with location of the four pressure rings $R 1-R 4$, c) cross section, d) experimental model, e) pressure taps locations and f) aerodynamic admittance tests.

$\sigma_{w} / U=1.1 \%$, respectively, with corresponding integral length scales of $L_{u}=0.124 \mathrm{~m}$ and $L_{w}=0.025 \mathrm{~m}$. These values are neg- ligible w.r.t. the actively generated turbulent component for the admittance tests. The wind tunnel tests are performed with low 

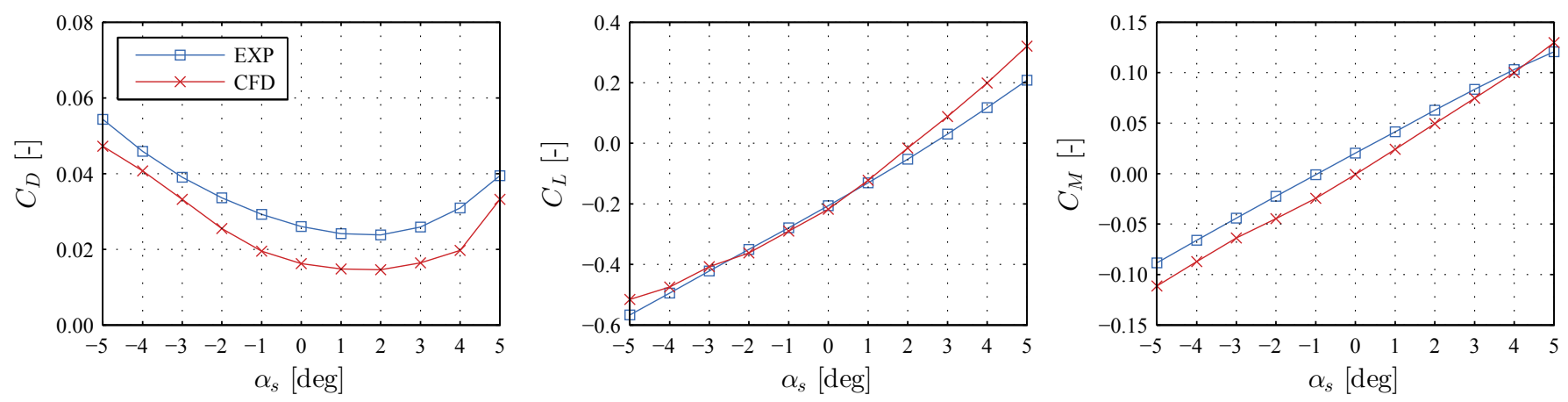

Figure 15: Static wind coefficients for the 3rd Bosphorus bridge for the CFD and experimental (EXP) model: drag (left), lift (center) and moment (right).

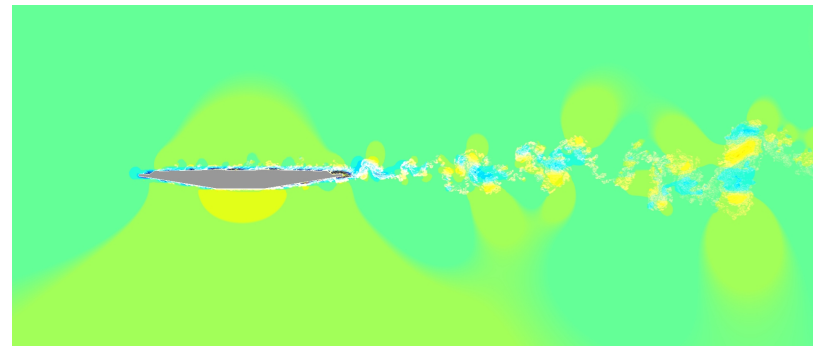

Figure 16: Instantaneous velocity field for laminar free-stream from a CFD analysis.

residual turbulence intensity since the aerodynamic admittance between the aerodynamic forces and the incoming wind turbulence is defined deterministically for each frequency. This approach allows to have a stronger control on the incoming turbulence both in terms of incoming turbulent wind velocity amplitude and phase shift between input and output.

\subsection{Static wind coefficients}

Initially, the static wind coefficients obtained using CFD simulations are compared to the experimental results. For each angular increment, the lift and moment coefficients are averaged for a minimum time period amounting to $s=50$. In the CFD simulations, the deck is discretized on 750 panels with a non-dimensional time-step of $\Delta s=0.0027$ and Reynolds number amounting to $\operatorname{Re}=7.78 \times 10^{5}$. The length of the CFD domain is set as $l_{d}=16 B$ with $\boldsymbol{x}_{s}=(6.5 B, 0)$ (cf. Fig. 1). An instantaneous velocity field and particle map of the section under laminar free-stream is depicted in Fig. 16. Figure 15 depicts the static wind coefficients, obtained from the CFD simulations and experimental tests, while Fig. 16 depicts an instantaneous velocity field under laminar free-stream. For the experimental tests, the static wind coefficients are obtained from the pressure taps positioned on the deck only, neglecting the fences and auxiliary equipment. The Reynolds number in the experimental tests amounts to $\mathrm{Re}=7.70 \times 10^{5}$. A slight overestimation is noted in the lift coefficient for positive angles; while there is an offset in the moment coefficient. The drag coefficient can be underestimated by the VPM, which has been also observed in other studies (Larsen and Walther, 1997). Although the aerodynamic forces from the experimental tests are obtained using the pressure taps only, the separation and reattachment point is still influenced by the auxiliary equipment. Taking this into account, the correspondence is considered to be fair.

\subsection{Aerodynamic admittance}

\subsubsection{Deterministic free-stream turbulence}

The section in both CFD and experimental analyses is subjected to vertical sinusoidal gusts. For the CFD analyses, the reduced reduced velocity is selected in the range of $2 \leq V_{r} \leq 50$ with prescribed gust amplitude of $w_{c t 0} / U=0.035$ and Reynolds number amounting to $\mathrm{Re}=7.78 \times 10^{5}$. Unlike for the flat plate case, here the distance between the wakes is taken as $R=1.5 B$ in order to avoid particles crossing the section. In the experiments, it is difficult to obtain exactly the prescribed reduced velocities and gust amplitude. Hence, the Reynolds number varies in the range of $4.9 \times 10^{5} \leq \operatorname{Re} \leq 1.16 \times 10^{6}$, while the gust amplitudes are in the range of $0.011 \leq w_{0} / U \leq 0.074$. As an example, Fig. 17 depicts the time-histories and their corresponding FFTs of the longitudinal and vertical velocity components. Despite the high frequency and additional longitudinal components, a clear sinusoidal harmonic is observed at $V_{r}=9.52$. The quality of this flow for this particular experimental time-history is $Q_{w} \approx 0.7$.

Figure 18 depicts an instantaneous particle map at the peak of a sinusoidal gust past the bridge deck at reduced velocity of $V_{r t}=16$. It is evident that the particles do not cross the section. Figure 19 depicts the fluctuating wind coefficients due to buffeting forces $C_{D b}^{*}, C_{L b}^{*}$ and $C_{M b}^{*}$ (i.e. the mean is subtracted), which are normalized w.r.t. gust amplitude $w_{0} / U$. The reduced velocity, Reynolds number and gust amplitudes are approximately similar for this case for the CFD analyses and experiments. Good correspondence can be observed, with a slight overestimation by the CFD model for the lift and moment. In case of the drag, the amplitudes at the prescribed reduced velocities correspond well, while additional low reduced velocity component can be observed from the experimental results. The drag force at $V_{r}=0.5 V_{r t}$ is higher for the experimental result$\mathrm{s}$ due to the higher contribution of the additional longitudinal velocity component. 

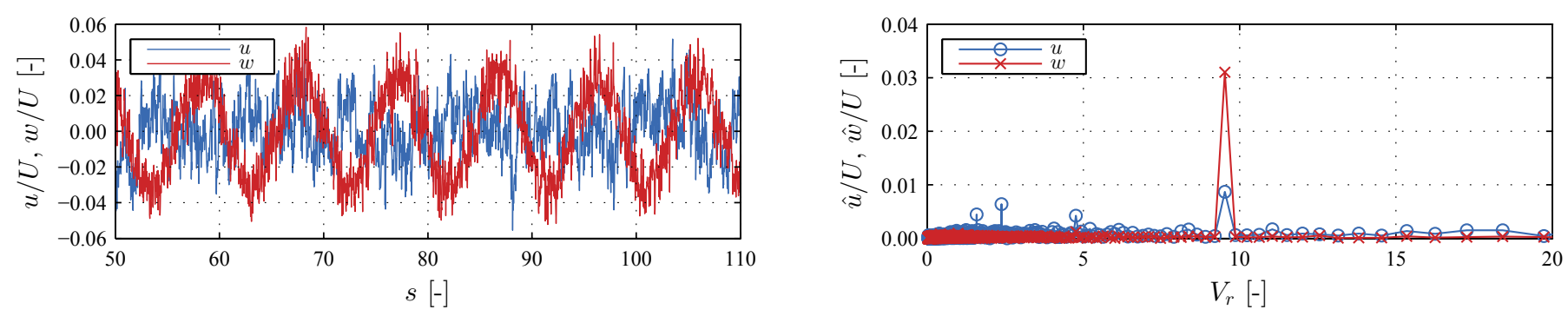

Figure 17: Time-histories of the fluctuating velocities (left) and corresponding FFTs (right) recorded from the cobra probe corresponding to pressure ring $R 3$ from experiments.

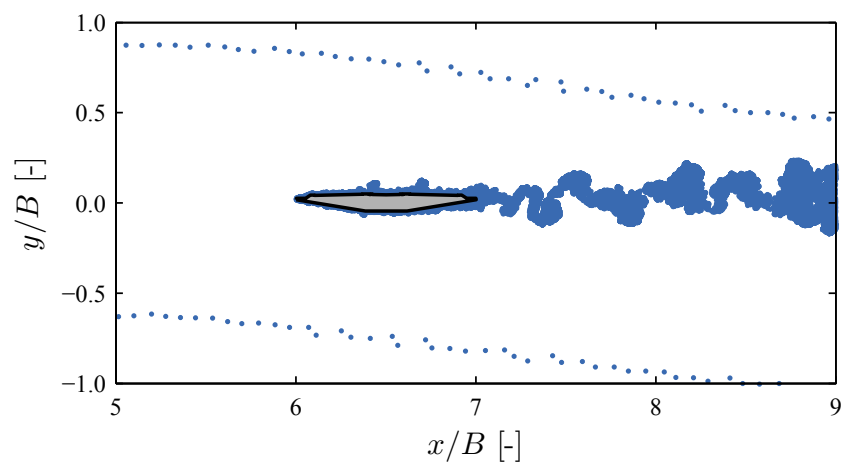

Figure 18: Instantaneous particle map for a sinusoidal vertical gust past the bridge deck at prescribed reduced velocity $V_{r t}=16$.

In Fig. 20, the aerodynamic admittance for the CFD and experimental analyses is depicted. The experimental results are for the inner pressure rings, R2 and R3 (cf. Fig 14). These two rings are selected in order to alleviate the end effects of the flow on the model. The aerodynamic admittance for the CFD model is computed for two setups. In the first setup (S1), the sinusoidal gust is tracked down at $B$ upwind of the leading edge (i.e. $\left.\boldsymbol{x}_{c}=(5 B, 0)\right)$, in a similar fashion as for the experimental results. For this setup, the optional step c) is not utilized (cf. Sec. 3.1). The aerodynamic admittance for the second setup (S2) is computed with a sinusoidal gust tracked at the position of the stiffness center (i.e. $\boldsymbol{x}_{c} \equiv \boldsymbol{x}_{s}=(6.5 B, 0)$ ), from a simulation without a section in the CFD domain. The optional step c) (cf. Sec. 3.1) is used for S2. In order obtain comparable results with the experiments for the imaginary value of the aerodynamic admittance, the sinusoidal gust is shifted for $t_{\text {shift }}=1.5 \mathrm{~B} / \mathrm{U}$ for $\mathrm{S} 2$. It is noted that, this does not influence the absolute value of the aerodynamic admittance. As it can be observed from the figure, the CFD results for the lift and moment correspond good with the experimental results for both real and imaginary components. In case of the drag aerodynamic admittance, the correspondence of the CFD results is better for R3, while some discrepancies are observed for R2. Obtaining the drag admittance implies division of the drag force with the difference $C_{D}^{\prime}-C_{L}$ (cf. Eqs. (5)), which value is very low. As seen from the static wind coefficients (cf. Fig. 15), the drag is highly nonlinear in the region of $\alpha_{s} \approx 0$ and the value of drag derivative $C_{D}^{\prime}$ is very small. The low frequency components in the experimental re- sults govern the drag force at certain reduced velocities as well, which means that the sensitivity of the drag due to vertical gusts is indeed very low. This explains partially the discrepancies for the drag admittance component. It is worthy to notice that, the drag aerodynamic admittance exceeds unity in both CFD and experimental results.

Two reasons seem plausible for difference between $\mathrm{S} 1$ and $\mathrm{S} 2$ for the CFD model. First, the distortion of the incoming gust due to the influence of the section, and second, the variation of the gust amplitude downstream. As discussed previously, the linear unsteady aerodynamic model does not take the effect of the section on the gust amplitudes into account. It is noteworthy to notice that in $\mathrm{S} 1$, the absolute value of the aerodynamic admittance tends faster towards unity than in the $\mathrm{S} 2$ case.

Furthermore, the aerodynamic admittance due to longitudinal gusts is determined only for the CFD model. All of the airfoils in the ATG used in the experimental tests are mechanically connected and oscillate in-phase. Hence, a pure longitudinal gust cannot be generated. For the CFD simulations, the prescribed longitudinal gust amplitude amounts to $u_{c t 0} / U=0.035$. Figure 22 depicts the aerodynamic admittance for the drag and moment forces. Although some alteration can be observed, the absolute value of the drag admittance is mostly unitary. The lift admittance is somewhat irregular w.r.t. Sears' function. The imaginary values for the drag and lift admittances are always negative, meaning that the forces lag behind the longitudinal gust for this particular point of tracking of the wind. This was not the case for the vertical gusts (cf. Fig 20). No significant difference between the two setups, $\mathrm{S} 1$ and $\mathrm{S} 2$, is observed.

The moment admittance due to longitudinal gusts is not obtained for this particular case. The reason for this is that the moment forces resulted in rather low values, which for most of the reduced velocity range are even lower than the vortex-shedding forces. This can be observed in the FFTs of the fluctuating coefficients in Fig. 22. Furthermore, the value of the moment coefficient $C_{M}$ at $\alpha_{s}=0$ is very close to zero (cf. Fig. 15); hence, the results are prone to high numerical uncertainties in the averaging procedure for the static wind coefficients. Therefore, the moment admittance resulted in very high values due to the small values in the denominator in Eq. (5) for moment aerodynamic admittance due to longitudinal gusts $\chi_{M u}$. As the moment coefficient $C_{M}$ at $\alpha_{s}=0$ is insignificant compared to the derivative $C_{M}^{\prime}$, the forces due to longitudinal gusts are negligible anyway. Nevertheless, for a different bridge deck with 



Figure 19: Normalized fluctuating wind coefficients due to buffeting forces (left) and their corresponding FFTs (right) for the CFD model $\left(\operatorname{Re}=7.78 \times 10^{5} ; w_{c 0} / U=0.040\right)$ and experimental $(\mathrm{EXP})$ model $\left(\operatorname{Re}=6.31 \times 10^{5} ; w_{c 0} / U=0.031\right)$ : drag (top); lift (center); and moment (bottom).

$C_{M} \not \approx 0$, obtaining the moment admittance due to longitudinal gusts is straightforward using the presented method.

\subsubsection{Random free-stream turbulence: Comparison}

Herein, the lift and moment aerodynamic admittance for vertical gusts obtained in the previous section are further compared with the CFD admittance for random free-stream turbulence by Rasmussen et al. (2010).

The random free-stream turbulence is generated in a similar fashion as Sec. 4.2.2. To maintain consistency with the vertical gust magnitudes and ensure linearity, isotropic turbulence intensity is set to $\sigma_{u} / U=\sigma_{w} / U=0.03$, while the length-scales are $L_{u}=4 B$ and $L_{w}=2 B$. Slightly higher particle release factor is selected compared to the flat plate study, amounting to $\Delta p=6$. The particle band height is set as $15 B$ and is positioned $6 B$ upstream of the leading edge. Identical coherence coefficients are used as in Sec. 4.2.2.

The aerodynamic admittance of the lift and moment is determined using Eqs. (34) based on Welch's PSD, in which no distinction can be made for the admittance due to vertical and horizontal gusts. Figure 23 depicts the absolute lift and moment aerodynamic admittances from CFD simulations for both, deterministic and random free-stream, and the experimental results for pressure rings R2 and R3. Setup S2 is used for both types of free-stream for the CFD simulations, i.e. the gust is tracked at $\boldsymbol{x}_{c}$ from a simulation without a section. Mostly, sim- ilar results can be observed for both random and deterministic free-stream for $V_{r} \gtrsim 5$. This shows that the same reasoning holds as for a flat plate (cf. Sec. 4.2.2), i.e. the admittance for both deterministic and random free-stream is same due to the $2 \mathrm{D}$ and linearity assumption. However, in case of bluffer decks and higher turbulence intensity, the superposition principle of the forces due to longitudinal and vertical fluctuating components may not apply. Thus, the capability of the conventional theoretical framework in Eqs. (4) may be exceeded as shown by Ma et al. (2013), where the 2D admittance was determined to be dependent on the turbulent characteristics of a single harmonic. This requires further investigations (Zhao and Ge, 2015). For lower reduced velocities ( $V_{r} \lesssim 5$ ) however, the numerical noise in the aerodynamic admittance based on random free-stream is more significant and there is a higher deviation w.r.t. experimental results than for the CFD results based on deterministic free-stream. This further proves the advantages discussed in Sec. 4.2.2.

\section{Critical remark: Strip assumption}

In this section, we discuss the the strip assumption, its role in bridge aerodynamics and the difference in the aerodynamic admittance for deterministic and random free-stream turbulence. 

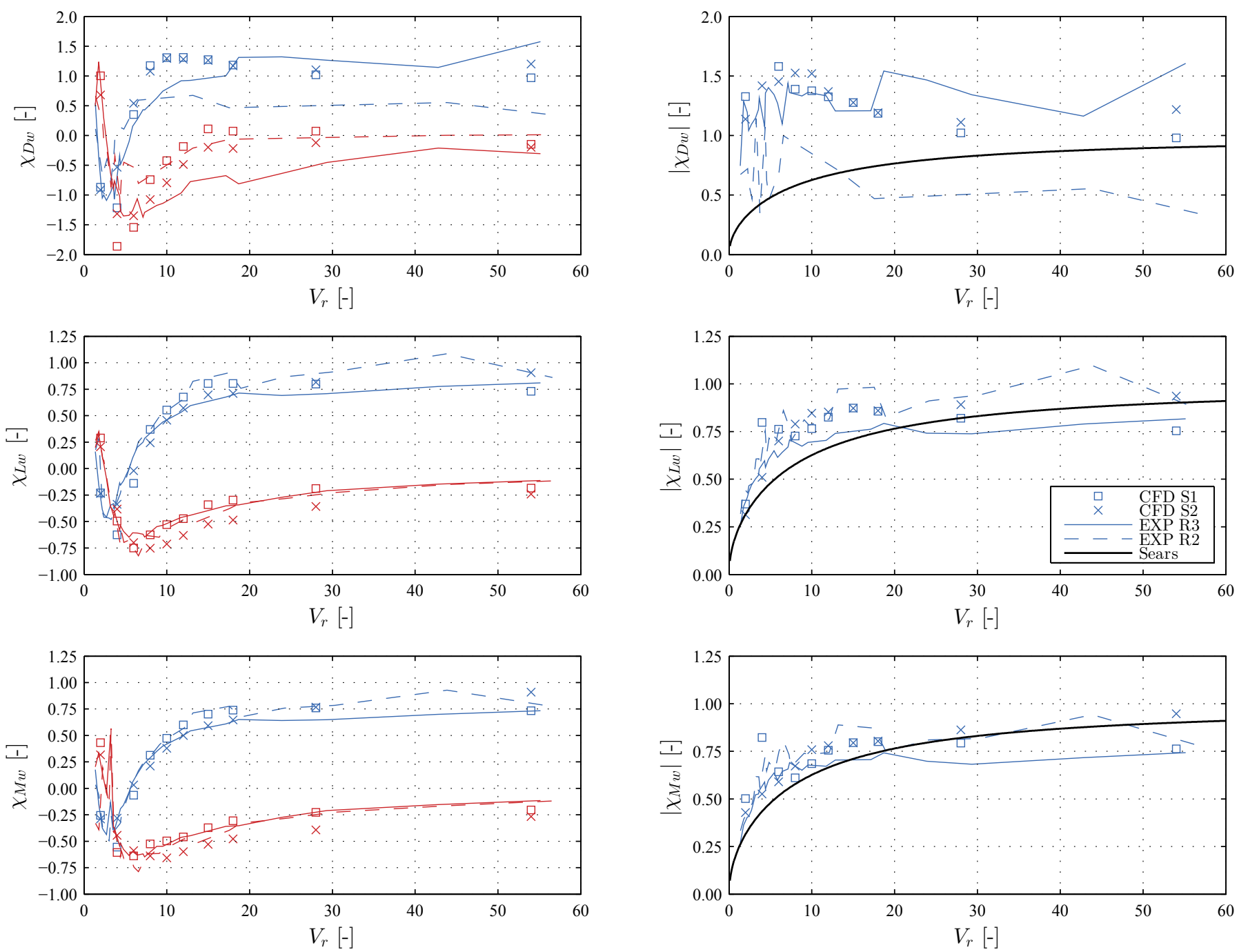

Figure 20: Aerodynamic admittance of the drag (top), lift (center) and moment (bottom) forces due to vertical sinusoidal gusts for the CFD and experimental (EXP) models: real (blue) and imaginary (red) parts (left); absolute values (right).

The aerodynamic model used herein for the buffeting forces, and hence, the aerodynamic admittance, is based on the 2D airfoil theory by Sears (1941), involving the strip assumption. The strip assumption implies that the aerodynamic admittance is not dependent on the span-wise wavenumber $k_{\text {span }}$ (i.e. onewavenumber admittance, $\chi=\chi(k, 0))$ and thus, the buffeting forces are uniformly distributed over the span of the strip. This is only true for fully correlated incoming gusts, which is not the case for grid or atmospheric random free-stream turbulence. To account for the 3D effects, Graham (1970) derived a twowavenumber aerodynamic admittance $\chi=\chi\left(k, k_{\text {span }}\right)$ for thin airfoils and solved it numerically. Approximated closed-form solutions for Graham's aerodynamic admittance were subsequently introduced by Mugridge (1971); Filotas (1969). Utilizing the general two-wavenumber admittance function, it can be shown why there is an increase in the span-wise correlation of the buffeting forces w.r.t. random free-stream turbulence (Massaro and Graham, 2015), which appeared in wind tunnel tests (Larose, 2002; Etkin, 1981). Recently, the two-wavenumber aerodynamic admittance was validated experimentally by $\mathrm{Li}$ et al. (2015).

Traditionally in bridge aerodynamics, the aerodynamic admittance under random free-stream turbulence in experiments is obtained simply by utilizing Eqs. (34) and averaging over the section length. This approach averages the buffeting forces, which are non-uniformly distributed over the span; hence, the one-wavenumber aerodynamic admittance can attain differen$t$ values for different span-to-width ratio of the experimental model. Therefore, the approach might not be appropriate for the linear unsteady model, described by Eqs. (4). Li et al. (2018) refer to the aerodynamic admittance obtain in such manner as 3D one-wavenumber aerodynamic admittance. To address the inconsistency in the force coherence in the 3D one-wavenumber aerodynamic admittance, many studies (cf. e.g. Davenport (1962)) directly include the increased buffeting force coherence by the joint-acceptance function. However, this is an empirical formulation that lack of physical meaning and consistency to interpret the 3D effects. Perhaps the most consistent approach 

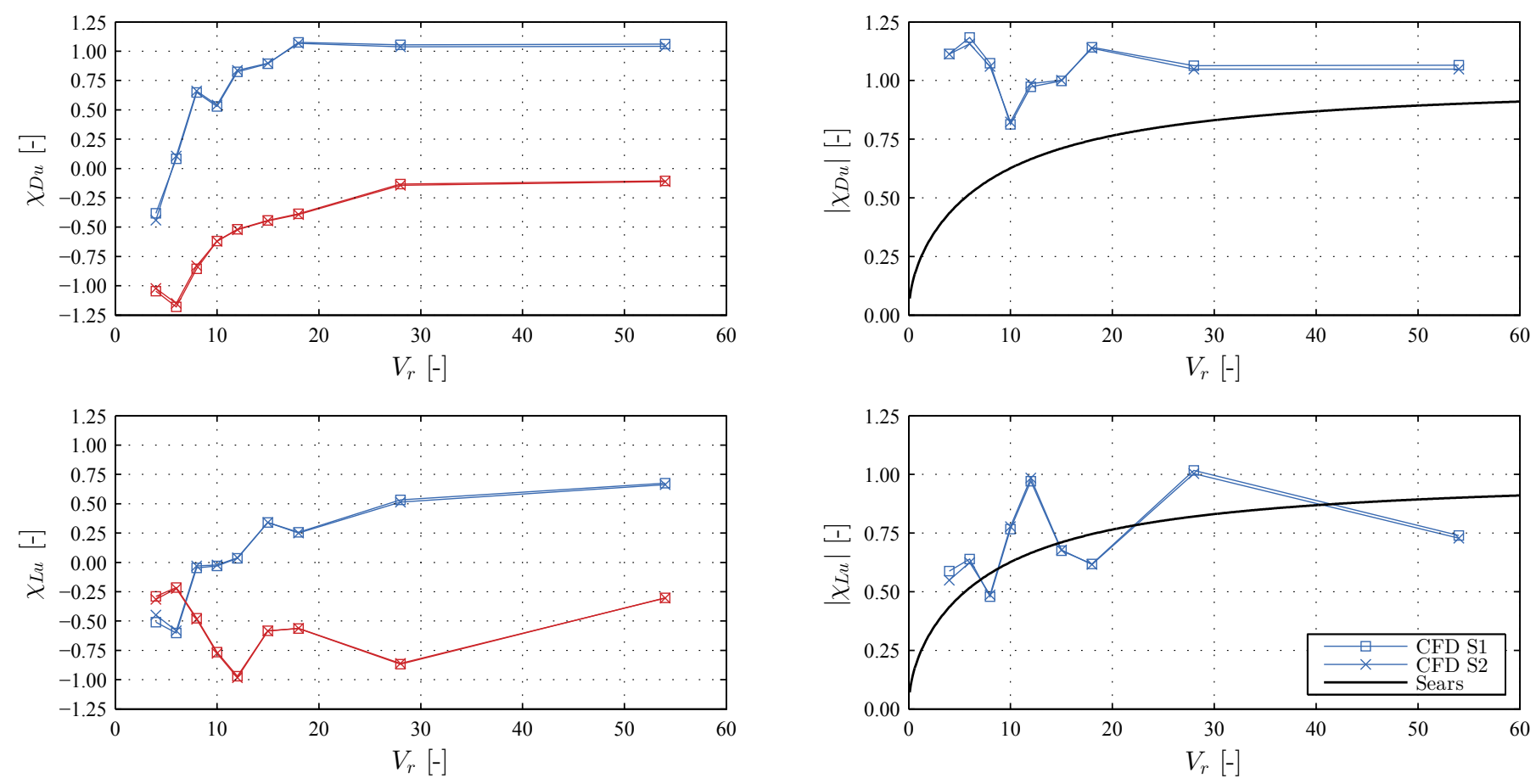

Figure 21: Aerodynamic admittance of the drag (top) and lift (bottom) forces due to longitudinal sinusoidal gusts for the CFD model: real (blue) and imaginary (red) parts (left); absolute values (right).

to account for the 3D effect into the buffeting forces is by utilizing the two-wavenumber admittance, obtained from random free-stream turbulence (Li et al., 2018). Nevertheless, this formulation of the buffeting forces is different than Eqs. (4) as it depends on both wavenumbers and a solution is only available in the frequency-domain.

It is well-established that the one-wavenumber aerodynamic admittance, obtained from deterministic free-stream turbulence, does not account for the 3D effects in the buffeting forces. However, when using the linear unsteady model (cf. Eqs. (4)), as it is per common practice in bridge aerodynamics, the onewavenumber aerodynamic admittance obtained from deterministic free-stream turbulence seems more appropriate than from random free-stream turbulence. In first case, the real and imaginary part of the aerodynamic admittance for an incoming sinusoidal gust can be clearly delineated at a particular frequency, otherwise not possible for random free-stream turbulence. As it was noted previously, a complex transfer function is required for the buffeting forces to be real signals in the time-domain (Kavrakov and Morgenthal, 2017). Moreover, in case of random free-stream turbulence, the effects of numerical uncertainty originating from the division of two PSDs (cf. Eqs. (34)), and the difficulties in wind similitude scaling for the low frequency components in experiments (Cigada et al., 2002) can unfavorably influence the results. A further argument for using an ATG to obtain the one-wavenumber aerodynamic admittance is that the strip assumption is generally valid for bodies with large span-to-width ratio, such as long-span bridges. This was shown in an analytical manner by Massaro and Graham (2015).

The application of the obtained one-wavenumber aerody- namic admittance could be further extended to obtain the $3 \mathrm{D}$ two-wavenumber aerodynamic admittance in a hybrid manner. This can be conducted by utilizing the closed-form of the 3D two-wave number aerodynamic admittance, $\chi\left(k, k_{\text {span }}\right)=\chi\left(\chi(k, 0), F^{3 \mathrm{D}}\left(k, k_{\text {span }}\right)\right)$, given by Li et al. $(2018)$, which includes the $3 \mathrm{D}$ effects separately by a correction factor $F^{3 \mathrm{D}}$. If this correction factor can be generalized for certain type of bridge decks, then the one-wavenumber aerodynamic admittance could be obtained from CFD simulations or experiments utilizing deterministic free-stream turbulence. However, this requires further validation. It is noted again that, the $3 \mathrm{D}$ effects are not considered in aerodynamic admittance obtained using the 2D VPM with random free-stream turbulence.

\section{Conclusion}

In this paper, we presented a novel method for the determination of the complex aerodynamic admittance using CFD. The method is based on the 2D VPM and deterministic free-stream turbulence in terms of sinusoidal gusts. Vortex particles are released at two locations, modeling the wakes of two fictitious airfoils that are oscillating upstream of the body. With this, longitudinal and vertical sinusoidal gusts were simulated for outof- and in-phase sinusoidal motion of the airfoils, respectively. A closed-form solution relating the gust and particles' circulation amplitudes was introduced, based on the analytical solution of a mathematical model by Stapountzis (1982).

The presented method was verified in terms of the flow field and Sears' aerodynamic admittance. The verification of the flow field was conducted based on a defined gust quality 

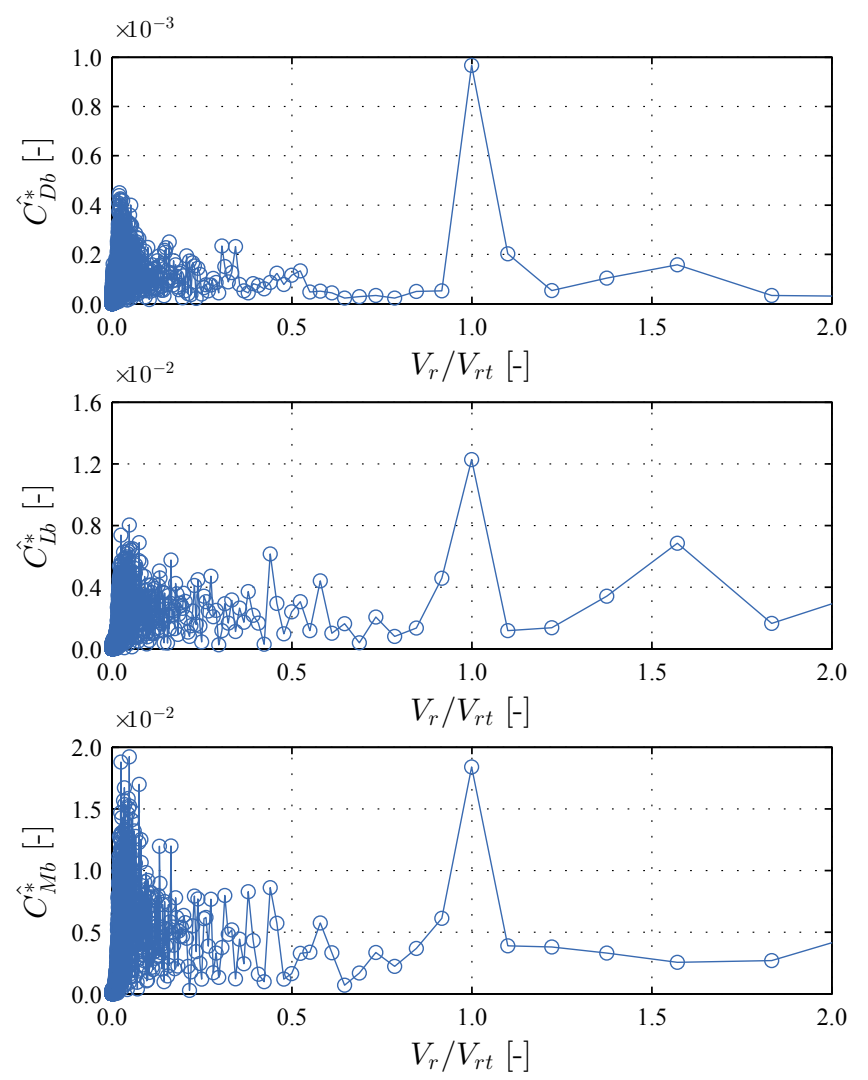

Figure 22: FFTs of the normalized fluctuating wind coefficients due to buffeting forces: drag (top), lift (center) and moment (bottom).

and prescribed gust amplitudes. It was shown that the gust is mostly sinusoidal with fairly good prediction of the gust amplitudes within the CFD domain. The results from the CFD analyses were found to be in very good agreement with the real and imaginary parts of the Sears' aerodynamic admittance. It was demonstrated that the presented method is more flexible than the method based on random free-stream turbulence, as the aerodynamic admittance can be determined in its complex form with reduced numerical uncertainty.

Finally, the results from the CFD analyses were validated with experimental results from wind tunnel tests for the $3^{\text {rd }}$ Bosporus bridge. Good correspondence was noted for both real and imaginary parts of the lift and moment aerodynamic admittance due to vertical gusts. The slight discrepancies in the drag aerodynamic admittance were attributed to the low values of the drag slope and lift coefficient at zero angle of attack. Moreover, the aerodynamic admittance was identified for the longitudinal fluctuating component from the CFD analyses. This presents the applicability of the presented method to determine the sixcomponent complex aerodynamic admittance.

In conclusion, the presented method is readily applicable for determination of the six-component one-wavenumber aerodynamic admittance for bridge decks. The outcome of this study is intended to be useful for researchers as well as practitioners. Further applications of the presented method could entail verification of the linear hypothesis, in terms of superposition of the self-excited and buffeting forces as well as of the individual buffeting force components due to longitudinal and vertical fluctuations. Moreover, the linear hypothesis could be also tested for varying gust intensity for both, random and deterministic, free-stream turbulence.

\section{Acknowledgments}

IK and GM gratefully acknowledge the support by the German Research Foundation (DFG) [Projects No. 43475018 and 329120866] throughout IK's studies and research stay in the Department of Mechanical Engineering at Politecnico di Milano.

\section{Appendix A. ATG Parametric studies}

Figures A.24, A.25, and A.26 are included in this section.

\section{References}

Abbas, T., Kavrakov, I., Morgenthal, G., 2017. Methods for flutter stability analysis of long-span bridges: a review. Proc Inst Civ Eng - Bridge Eng 170, 271-310. doi:10.1680/jbren.15.00039.

Argentini, T., Rocchi, D., Muggisaca, S., Zasso, A., 2012. Cross-sectional distribution versus integrated coefficients of flutter derivatives and aerodynamic admittances identified with surface pressure measurement. J Wind Eng Ind Aerodyn 104-106, 152-158.

Bisplinghoff, R.L., Ashley, H., Halfman, R.L., 1996. Aeroelasticity. Dover ed., Dover Publications, INC.

Blasius, H., 1950. The boundary layers in fluids with little friction. Technical Memorandum 1256. NACA. Washington, USA.

Bruno, L., Tubino, F., Solari, G., 2004. Aerodynamic admittance functions of bridge deck sections by CWE, in: IN-VENTO, Reggio Calabria, Italy.

Cao, S.Y., Nishi, A., Kikugawac, H., Matsuda, Y., 2002. Reproduction of wind velocity history in a multiple fan wind tunnel. J Wind Eng Ind Aerodyn 90, 1719-1729. doi:10.1016/S0167-6105(02)00282-9.

Chawdhury, S., Milani, D., Morgenthal, G., 2018. Modeling of pulsating incoming flow using vortex particle methods to investigate the performance of flutter-based energy harvesters. Comp Struct 209, 130-149. doi:10.1016/j.compstruc.2018.08.008.

Chen, X., Kareem, A., 2002. Advances in modeling of aerodynamic forces on bridge decks. J Eng Mech 128, 1193-1205. doi:10.1061/(ASCE)07339399(2002)128:11(1193).

Chorin, A., 1973. Numerical study of slightly viscous flow. J Fluid Mech 27, 785-796. doi:10.1017/S0022112073002016.

Cigada, A., Diana, G., Zappa, E., 2002. On the response of a bridge deck to turbulent wind: a new approach. J Wind Eng Ind Aerodyn 90, 1173-1192. doi:10.1016/S0167-6105(02)00230-1.

Cottet, G., Koumoutsakos, P., 2000. Vortex methods: Theory and Practice. Cambridge University Press.

Davenport, A.G., 1962. The response of slender, line-like structures to a gusty wind. Proc Inst Civ Eng 23, 389-408. doi:10.1680/iicep.1962.10876.

Diana, G., Bruni, S., Cigada, A., Zappa, E., 2002. Complex aerodynamic admittance function role in buffeting response of a bridge deck. J Wind Eng Ind Aerodyn 90, 2057-2072. doi:10.1016/S0167-6105(02)00321-5.

Diana, G., Rocchi, D., Argentini, T., 2013. An experimental validation of band superposition model of the aerodynamic forces acting on a multi-box deck sections. J Wind Eng Ind Aerodyn 113, 40-58. doi:10.1016/j.jweia.2012.12.005.

Etkin, B., 1981. Turbulent wind and its effect on flight. J Aircr 18, 327-35. doi:10.2514/3.57498.

Filotas, L.T., 1969. Theory of airfoil response in a gusty atmosphere. Part I Aerodynamic transfer function. Technical Report 139. UTIAS. Washington, USA. 

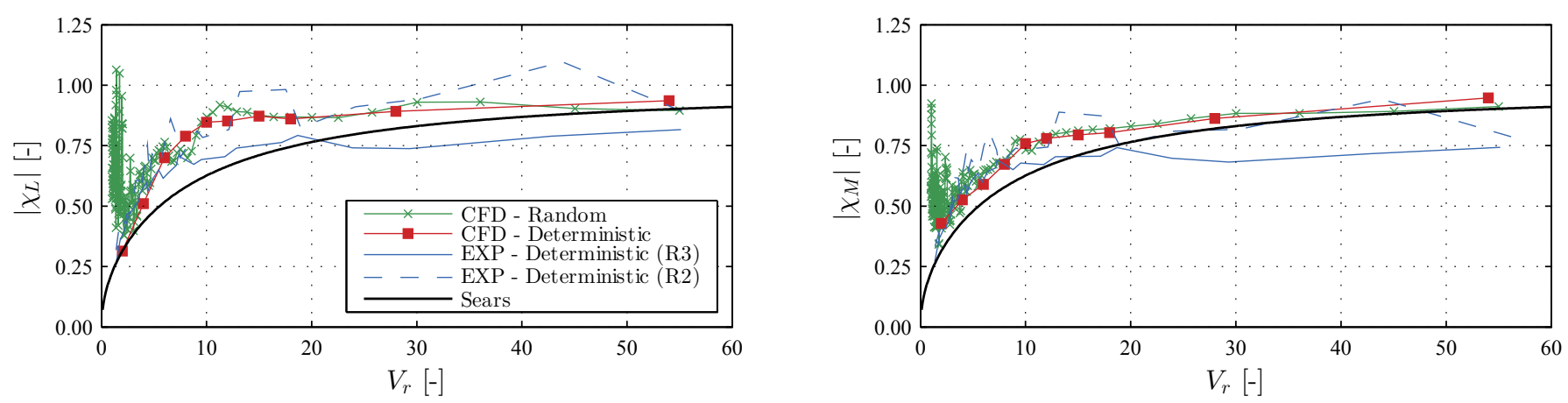

Figure 23: Aerodynamic admittance of the lift (left) and moment (right) forces the CFD and experimental (EXP) models. The CFD results based on both, deterministic and random free-stream turbulence, are for setup S2, i.e. the gust is tracked at the stiffness center from a simulation without a section.
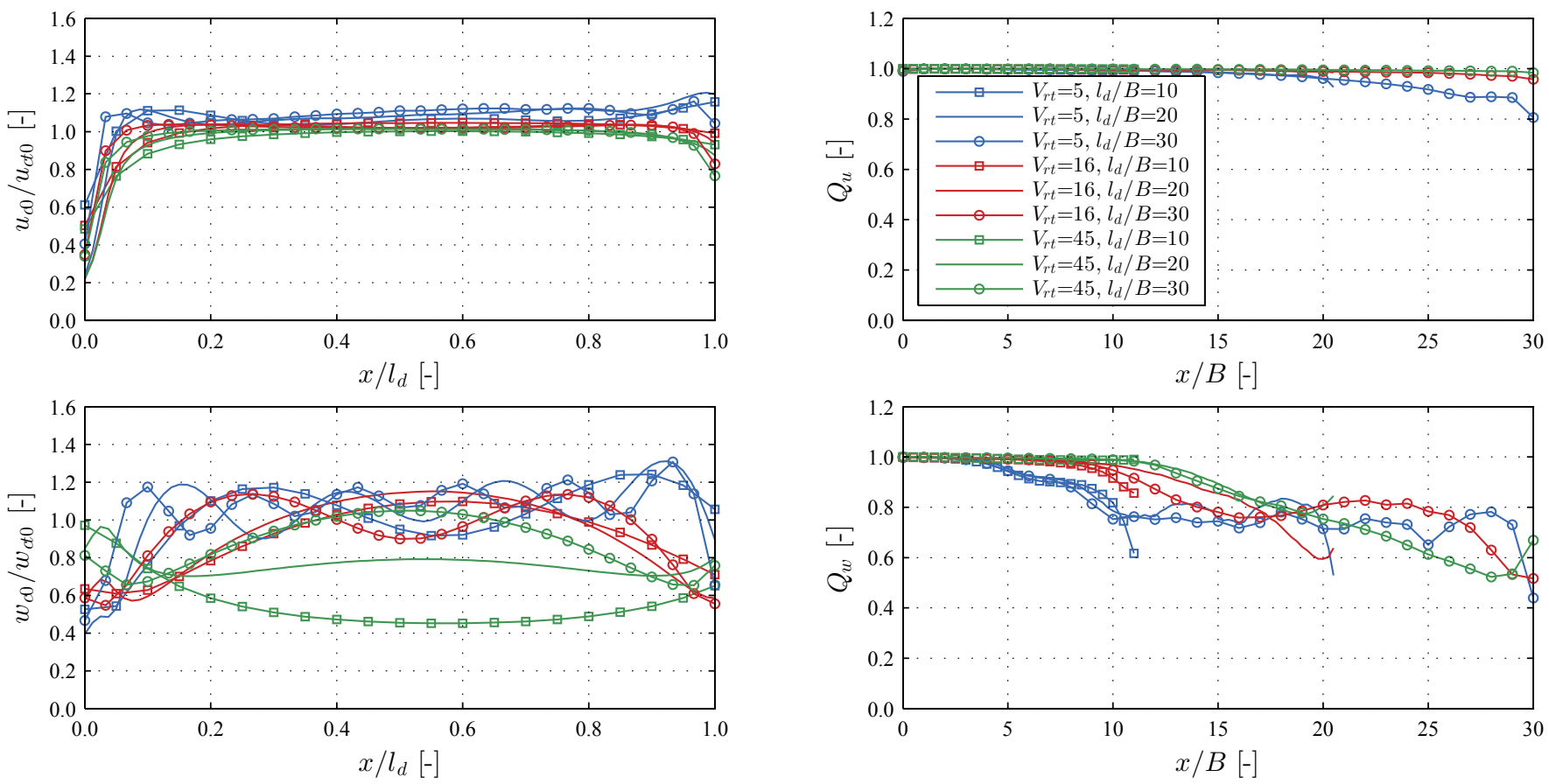

Figure A.24: Influence of the summation length $l_{d}$ on the relative gust amplitude (left) and quality (right) for airfoils oscillating out-of-phase (top) and in-phase (bottom) along the centerline $y=0$.

Ge, Y., Xiang, H.F., 2008. Computational models and methods for aerodynamic flutter of long-span bridges. J Wind Eng Ind Aerodyn 96, 1912-1924. doi:10.1016/j.jweia.2008.02.017.

Graham, J.M.R., 1970. Lifting surface theory for the problem of an arbitrarily yawed gust incident of a thin aerofoil in incompressible flow. Aeronaut Quart 18, 182-198. doi:10.1017/S0001925900005357.

Gu, M., Qin, X.R., 2004. Direct identification of flutter derivatives and aerodynamic admittances of bridge decks. Eng Struct 26, 2161-2172. doi:10.1016/j.engstruct.2004.07.015.

Han, Y., Chen, Z., Hua, X.G., 2010. New estimation methodology of six complex aerodynamic admittance functions. Wind and Struct 13, 293-307. doi:10.12989/was.2010.13.3.293.

Harding, S., Bryden, I.G., 2012. Generating controllable velocity fluctuations using twin oscillating hydrofoils. J Fluid Mech 713, 150-158. doi:10.1017/jfm.2012.442

Harding, S., Payne, G.S., Bryden, I.G., 2014. Generating controllable velocity fluctuations using twin oscillating hydrofoils: experimental validation. J Fluid Mech 713, 113-123. doi:10.1017/jfm.2014.257.

Hejlesen, M.M., Rasmussen, J.T., Larsen, A., Walther, J.H., 2015. On estimat- ing aerodynamic admittance of bridge section by mesh-free vortex method. J Wind Eng Ind Aerodyn 146, 117-127. doi:10.1016/j.jweia.2015.08.003.

Helgedagsurd, T.A., Bazlives, Y., Mathisen, K.M., Øsieth, O., 2019. Modeling and simulation of bridge-section buffeting response in turbulent flow. Math Models Methods Appl Sci 29, 939-966. doi:10.1142/S0218202519410045.

Hockney, R., Eastwood, J., 1988. Computer Simulation Using Particles. Taylor $\&$ Francis.

Jancauskas, E.D., Melbourne, W.H., 1986. The aerodynamic admittance of two-dimensional rectangular section cylinders in smooth flow. J Wind Eng Ind Aerodyn 23, 395-408. doi:10.1016/0167-6105(86)90057-7.

Kareem, A., Wu, T., 2013. Wind-induced effects on bluff bodies in turbulent flows: nonstationary, non-Gaussian and nonlinear features. J Wind Eng Ind Aerodyn 122, 21-37. doi:10.1016/j.jweia.2013.06.002.

Kavrakov, I., Legatiuk, D., Gürlebeck, K., Morgenthal, G., 2019. A categorical perspective towards aerodynamic models for aeroelastic analyses of bridge decks. R Soc Open Sci 6, 181848. doi:10.1098/rsos.181848.

Kavrakov, I., Morgenthal, G., 2017. A comparative assessment of aerodynamic models for buffeting and flutter of long-span bridges. Eng 3, 823-838. doi:10.1016/j.eng.2017.11.008 

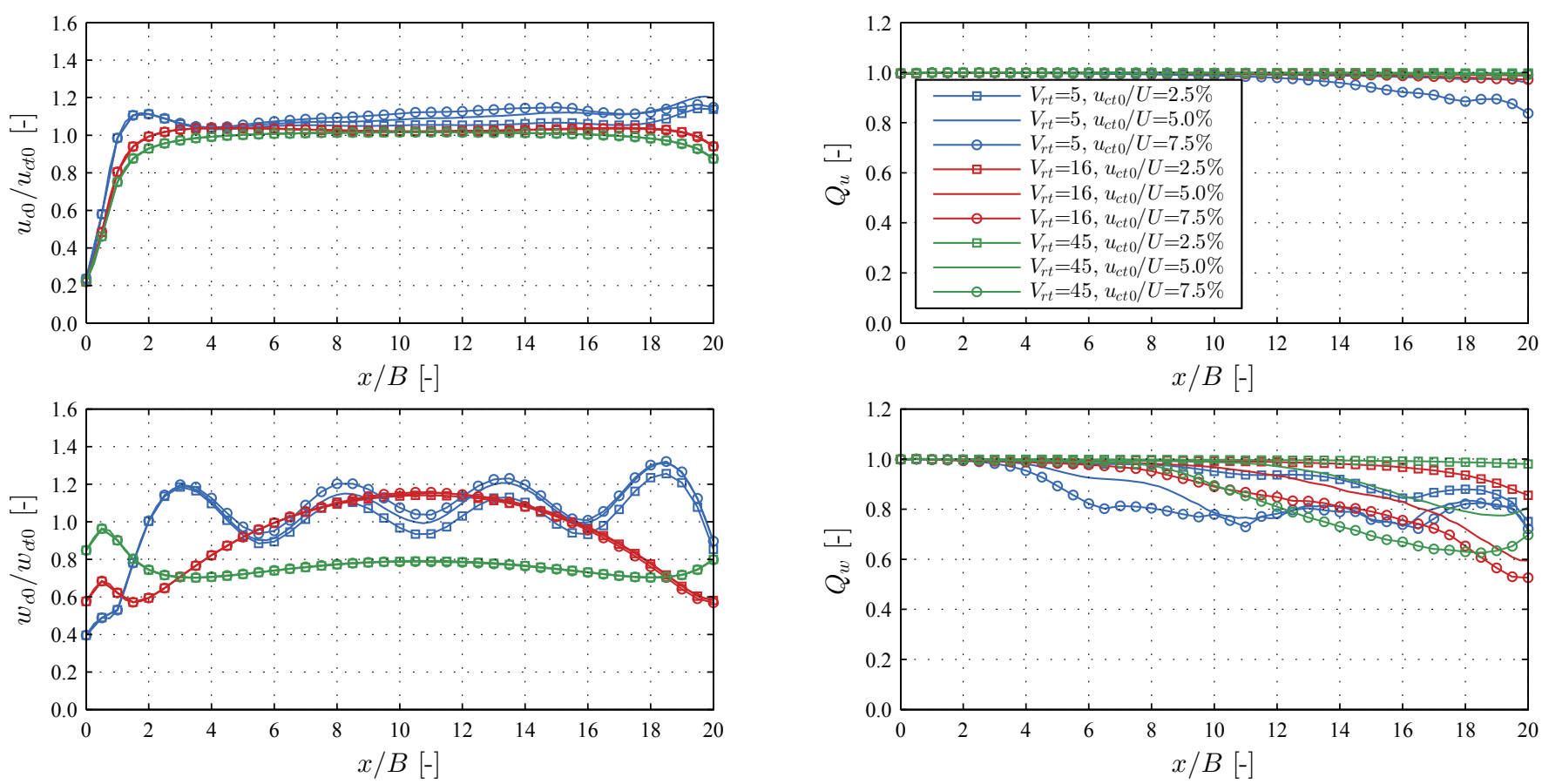

Figure A.25: Influence of the absolute gust intensity on the relative gust amplitude (left) and quality (right) for airfoils oscillating out-of-phase (top) and in-phase (bottom) along the centerline $y=0$.
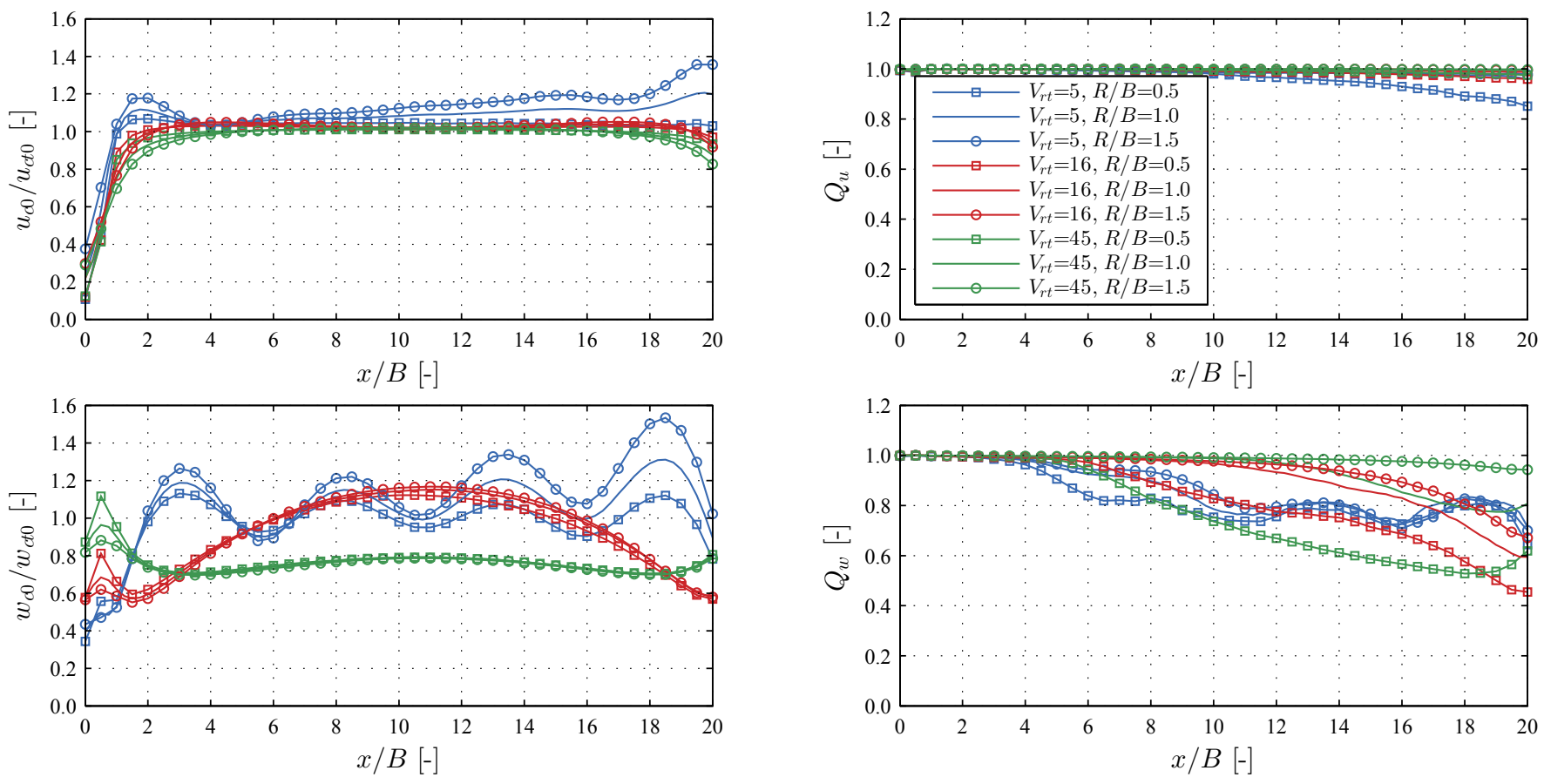

Figure A.26: Influence of the airfoil distance $R$ on the relative gust amplitude (left) and quality (right) for airfoils oscillating out-of-phase (top) and in-phase (bottom) along the centerline $y=0$.

Kavrakov, I., Morgenthal, G., 2018a. Aeroelastic analyses of bridges using a Pseudo-3D vortex method and velocity-based turbulence generation. Eng Struct 176, 825-839. doi:10.1016/j.engstruct.2018.08.093.

Kavrakov, I., Morgenthal, G., 2018b. A synergistic study of a CFD and semianalytical models for aeroelastic analysis of bridges in turbulent wind conditions. J Fluid Struct 82, 59-85. doi:10.1016/j.jfluidstructs.2018.06.013.

Larose, L., 2002. The dynamic action of gusty winds on long-span bridges.
Technical Report BYG-DTU R-029. DTU. Lyngby, Denmark.

Larsen, A., Walther, J.H., 1997. Aeroelastic analysis of bridge girder sections based on discrete vortex simulations. J Wind Eng Ind Aerodyn 67\&68, 253265. doi:10.1016/S0167-6105(97)00077-9.

Li, S., Li, M., Larose, G.L., 2018. Aerodynamic admittance of streamlined bridge decks. J Fluid Struct 78, 1-23. doi:10.1016/j.jfluidstructs.2017.12.014. 
Li, S., Li, M., Liao, H., 2015. The lift on an airfoil in grid-generated turbulence. J Fluid Mech 771, 16-35. doi:10.1017/jfm.2015.162.

Ma, T.T., Zhao, L., Cao, S.Y., Ge, Y.J., Miyagi, H., 2013. Investigation$\mathrm{s}$ of aerodynamic effects on streamlined box girder using two-dimensional actively-controlled oncoming flow. J Wind Eng Ind Aerodyn 122, 118-129. doi:10.1016/j.jweia.2013.07.011.

Massaro, M., Graham, J., 2015. The effect of three-dimensionality on the aerodynamic admittance of thin sections in free stream turbulence. J Fluids Struct 57, 81-90. doi:10.1016/j.jfluidstructs.2015.05.012.

Matsuda, K., Hikami, Y., Fujiwara, T., Moriyama, A., 1999. Aerodynamic admittance and the 'strip theory' for horizontal buffeting forces on bridge deck. J Wind Eng Ind Aerodyn 83, 337-346. doi:10.1016/S0167-6105(99)000835 .

Morgenthal, G., Corriols, A., Bendig, B., 2014. A GPU-accelerated pseudo$3 \mathrm{D}$ vortex method for aerodynamic analysis. J Wind Eng Ind Aerodyn 125, 69-80. doi:10.1016/j.jweia.2013.12.002.

Morgenthal, G., Walther, J., 2007. An immersed interface method for the Vortex-In-Cell algorithm. Comput Struct 85, 712-726. doi:10.1016/j.compstruc.2007.01.020.

Mugridge, B.D., 1971. Gust loading on a thin airfoil. Aeronaut Quart 22, 301-310. doi:10.1017/S0001925900005849.

Prendergast, J., 2007. Simulation of 2D unsteady wind by a vortex method. Ph.D. thesis. Cambridge University.

Rasmussen, J.T., Hejlesen, M.M., Larsen, A., Walther, J.H., 2010 Discrete vortex method simulations of the aerodynamic admittance in bridge aerodynamics. J Wind Eng Ind Aerodyn 99, 776-785. doi:10.1016/j.jweia.2010.06.011.

Sears, W.R., 1941. Some aspects of non-stationary airfoil theory and its practical application. J AIAA 8, 104-108. doi:10.2514/8.10655.

Solari, G., Piccardo, G., 2000. Probabilistic 3-D turbulence modeling for gust buffeting of structures. Prob Eng Mech 16, 73-86. doi:10.1016/S02668920(00)00010-2.

Stapountzis, H., 1978. Lift forces on cylindrical bodies in unsteady flow. Ph.D thesis. Imperial College London.

Stapountzis, H., 1982. An oscillating rig for the generation of sinusoidal flows J Phys E Sci Instrum 15, 1173-1176.

Stapountzis, H., Graham, J.M.R., 1982. The unsteady lift on bluff cylindrical bodies in unsteady flow. Aeronaut Quat 3, 219-236. doi:doi:10.1017/S0001925900009434.

Tang, D.M., Cizmas, P.G.A., Dowel, E.H., 1996. Experiments and analysis for a gust generator in a wind tunnel. J Aircr 33, 139-147. doi:10.2514/3.46914.

Turbelin, G., Gibert, R.J., 2001. CFD calculation of indicial lift responses for bluff bodies. Wind Struct 5, 245-256. doi:10.12989/was.2002.5.2_3_4.245.

Uejima, H., Kuroda, S., Kobayashi, H., 2008. Estimation of aerodynamic admittance by numerical computation, in: BBAA VI International Colloquium, Milan, Italy.

Walther, J., Larsen, A., 1997. Two dimensional discrete vortex method for application to bluff body aerodynamics. J Wind Eng Ind Aerodyn 67\&68, 183-193. doi:10.1016/S0167-6105(97)00072-X.

Wu, J.C., 1976. Numerical boundary conditions for viscous flow problems. AIAA 14, 1042-1049. doi:10.2514/3.61439.

Zhao, L., Ge, Y.J., 2015. Cross-spectral recognition method of bridge deck aerodynamic admittance function. Earthq Eng Eng Vib 14, 595-609. doi:10.1007/s11803-015-0048-8. 